\title{
Extinction and stationary distribution of a stochastic SIQR epidemic model with demographics and non-monotone incidence rate on scale-free networks
}

\author{
Yuncheng $\mathrm{Xu}^{1}$ (D) Xiaojun Sun ${ }^{2} \cdot \mathrm{Hua} \mathrm{Hu^{1 }}$
}

Received: 14 July 2021 / Revised: 18 September 2021 / Accepted: 6 October 2021 /

Published online: 24 November 2021

(c) Korean Society for Informatics and Computational Applied Mathematics 2021

\begin{abstract}
By taking full consideration of contact heterogeneity of individuals, quarantine measures, demographics, information transmission and random environments, we present a stochastic SIQR epidemic model with demographics and non-monotone incidence rate on scale-free networks, which introduces stochastic perturbations to death rate. The formula of the basic reproduction number of the deterministic model is obtained by utilizing the existence of the endemic equilibrium. Next, we define a stopping time, then the existence of a unique global positive solution for the stochastic model is proved by constructing appropriate Lyapunov function to demonstrate the stopping time is infinite. In addition, we also manifest sufficient conditions for diseases extinction and the existence of ergodic stationary distribution by constructing appropriate stochastic Lyapunov functions. At last, numerical simulations illustrate the analytical results.
\end{abstract}

Keywords Stochastic SIQR epidemic model · Non-monotone incidence rate · Extinction $\cdot$ Stationary distribution $\cdot$ Scale-free networks

Mathematics Subject Classification $60 \mathrm{H} 10 \cdot 34 \mathrm{H} 15 \cdot 92 \mathrm{D} 30$

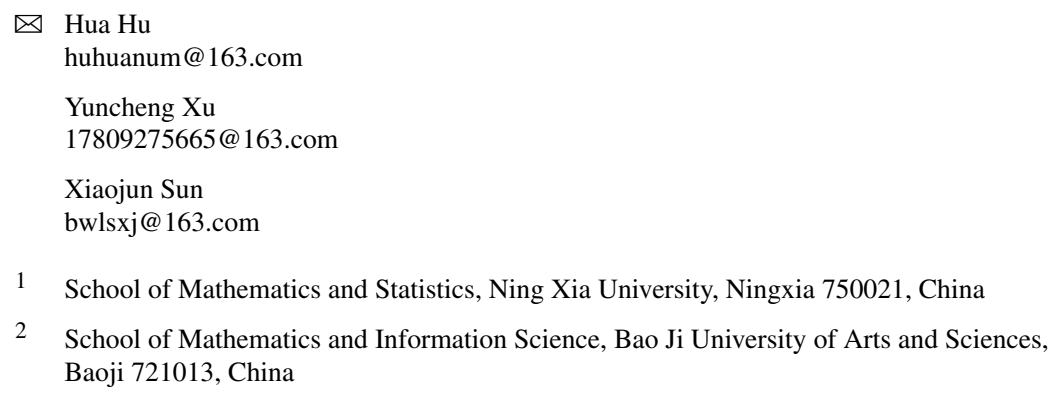




\section{Introduction}

Infectious diseases bring human not only physical pain, but also the loss of property and serious damage environment. Along with the acceleration of globalization and the development of technology, the speed of cross regional transmission of infectious diseases is faster than at any time in history, so it is becoming more and more important to research the dynamic behavior of infectious diseases. Although traditional compartment models under the assumption of homogeneous mixing have been widely researched for a long time [1-5], obviously, it cannot adequately reflect the realistic characteristics of diseases transmission, such as the transmission pattern of Severe Acute Respiratory Syndrome (SARS) and Acquired Immunodeficiency Syndrome (AIDS) are heterogeneous [6, 7]. However, since the scale-free network was proposed by Barabási and Albert [8], in which the probability of $P(k)$ for any node with $k$ links to other nodes is distributed follow the power law $P(k)=C k^{-\gamma}(2<\gamma \leq 3)$, people find that most of the complex systems in the real world exhibit characteristics consistent with scale-free networks, such as the World Wide Web, the Internet, the human contact network and so on $[9,10]$. Accordingly, for further exploring of epidemic dynamics in real world, there have captured an increasingly amount of interest in studying the epidemic dynamic on complex networks [11-17]. Pastor-Satorras and Vespignani [12] discussed a SIS model on scale-free networks, a striking conclusion was obtained that the threshold approaching zero for sufficiently large scale-free networks, which changes traditional threshold theory and commendably explains the reason why computer viruses exist for a long time and cannot eradicate. As is known to all, quarantine measure plays an appreciable role in controlling the spread of diseases. To examine the effect of quarantine measure on diseases spreading, Li et al. [14] analyzed a SIQRS epidemic model on scale-free networks and found that the network topology and quarantine measures have significant effects on the threshold. But they did not take into account demographics, indeed, affected by population migration, resource constraints and diseases threats, the population size will change when the disease is prevalence for a long time. Accordingly, Huang et al. [15] studied the effects of demographics and vaccination on the dynamic behavior of infectious diseases. What is more, the incidence rate would decrease at high infective levels, because those who are informed the epidemic news would take protection measures (wearing masks and isolating themselves at home). Xiao and Ruan [18] described this phenomenon by introducing a non-monotone incidence rate: $g(I) S=\frac{k I S}{1+\alpha I^{2}}$, inspired by the work, Li [19] introduced this non-monotone incidence rate to the network-based SIS epidemic model and entertainingly, the functional form of the non-monotone incidence rate has no effect on threshold.

Generally speaking, there are various random factors in the transmission of infectious diseases, white noise is usually used to describe the random variability of environment. Therefore, there have captured an increasing amount of interest in studying stochastic epidemic models [20-26], the existence of ergodic stationary distribution and diseases extinction analyses are effective methods to discuss the spreading dynamics of stochastic epidemic models. Liu et al. studied a stochastic SEIR epidemic model with standard incidence and provided sufficient conditions for the existence of ergodic stationary distribution and diseases extinction [21]. Zhang et al. formulated a 
network-based stochastic SEIR epidemic model by perturbing transmission rate and conversion rate, the influences of human awareness and migration on the dynamic of diseases are analyzed [22]. And Zhao et al. considered the effects of quarantine measures and built a stochastic SIQS epidemic model on scale-free networks, and sufficient conditions for persistence and extinction are established [23]. To the best of our knowledge, there are relatively few works to study the extinction and stationary distribution of stochastic epidemic models with quarantine measures, demographics and information transmission on scale-free networks.

The organization of the paper is as follows: In Sect. 2, a stochastic SIQR epidemic model with demographics and non-monotone incidence rate on scale-free networks is presented. In Sect. 3, the existence of equilibrium and the formula of the basic reproduction number is obtained. In Sect. 4, we provide some necessary lemmas. In Sect. 5, existence and uniqueness of the positive solution is investigated. In Sect. 6, we establish sufficient conditions for diseases extinction. What is more, we demonstrate that stochastic model has an ergodic stationary distribution under certain conditions in Sect. 7. Numerical simulations are given to illustrate the theoretical analysis in Sect. 8 , and then Sect. 9 concludes the full text and prospects the next work.

\section{Model formulation}

In this section, motivated by references $[14,15,19]$, we take full consideration of contact heterogeneity of individuals, quarantine measures, demographics and information transmission to present SIQR epidemic model with demographics and non-monotone incidence rate on scale-free networks. Every individual has one of the four epidemiological states: Susceptible (S), Infected (I), Quarantined (Q) and Removed (R), the flow diagram of the epidemic model is given in Fig. 1.

Considering the contact heterogeneity in population, then the dynamic mean-field equations can be generalized as below:

$$
\left\{\begin{array}{l}
\frac{d S_{k}(t)}{d t}=-\beta k S_{k}(t) \frac{\Theta(t)}{1+b \Theta^{2}(t)}-\alpha S_{k}(t)-\mu S_{k}(t)+\mu, \\
\frac{d I_{k}(t)}{d t}=\beta k S_{k}(t) \frac{\Theta(t)}{1+b \Theta^{2}(t)}-v I_{k}(t)-\gamma I_{k}(t)-\mu I_{k}(t)-\lambda I_{k}(t), \\
\frac{d Q_{k}(t)}{d t}=v I_{k}(t)-\varepsilon Q_{k}(t)-\mu Q_{k}(t)-\lambda Q_{k}(t), \\
\frac{d R_{k}(t)}{d t}=\alpha S_{k}(t)+\gamma I_{k}(t)+\varepsilon Q_{k}(t)-\mu R_{k}(t) .
\end{array}\right.
$$

Fig. 1 The flow diagram of the SIQR mode

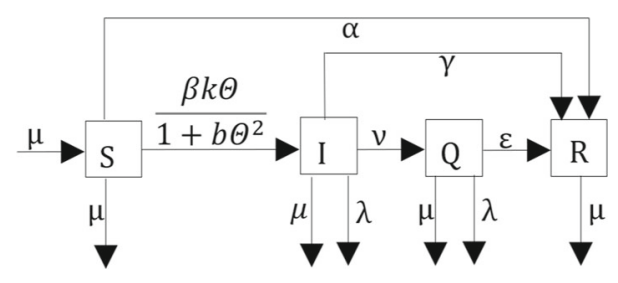


Table 1 Parameters of the model

\begin{tabular}{ll}
\hline Parameter & Biological significance \\
\hline$\beta$ & Transmission rate per contact between a susceptible and an infectious individual \\
$\alpha$ & The rate of susceptible individuals are vaccinated \\
$\mu$ & Birth rate, natural death rate \\
$v$ & Quarantine rate of infective individual \\
$\gamma$ & Cure rate of infective individuals \\
$\lambda$ & Mortality from diseases \\
$\varepsilon$ & Cure rate of quarantined individuals \\
\hline
\end{tabular}

Here $S_{k}(t), I_{k}(t), Q_{k}(t)$ and $R_{k}(t)$ are used to represent the density of Susceptible, Infected, Quarantined and Removed individuals of connectivity $k$ at time $t$ respectively. The parameters $\beta, \alpha, \mu, v, \gamma, \lambda$ and $\varepsilon$ are considered to be positive constants, and their biological significance is shown in Table $1 . \Theta(t)$ represents the probability that the susceptible individual is connected to an infected individual, which satisfies $\Theta(t)=$ $\frac{1}{\langle k\rangle} \sum_{k=1}^{n} k P(k) I_{k}(t)$, where $\langle k\rangle=\sum_{k=1}^{n} k P(k)$ is the average degree, $P(k)$ is the degree distribution, and the positive integer $n$ is the maximum degree of the network.

Because random disturbances are all the time and everywhere. Inspired by references [21-23], we introduce the effect about white noise of natural death rate and mortality from diseases of the deterministic model (1) in the stochastic environments, i.e. $\mu \rightarrow \mu+\sigma_{1} \dot{B}_{1}(t), \lambda \rightarrow \lambda+\sigma_{2} \dot{B}_{2}(t)$, then the corresponding random model is as follows:

$$
\left\{\begin{aligned}
d S_{k}(t) & =\left[-\beta k S_{k}(t) \frac{\Theta(t)}{1+b \Theta^{2}(t)}-\alpha S_{k}(t)-\mu S_{k}(t)+\mu\right] d t-\sigma_{1} S_{k}(t) d B_{1}(t) \\
d I_{k}(t) & =\left[\beta k S_{k}(t) \frac{\Theta(t)}{1+b \Theta^{2}(t)}-v I_{k}(t)-\gamma I_{k}(t)-\mu I_{k}(t)-\lambda I_{k}(t)\right] d t \\
& -\sigma_{1} I_{k}(t) d B_{1}(t)-\sigma_{2} I_{k}(t) d B_{2}(t) \\
d Q_{k}(t) & =\left[v I_{k}(t)-\varepsilon Q_{k}(t)-\mu Q_{k}(t)-\lambda Q_{k}(t)\right] d t-\sigma_{1} Q_{k}(t) d B_{1}(t) \\
& -\sigma_{2} Q_{k}(t) d B_{2}(t) \\
d R_{k}(t) & =\left[\alpha S_{k}(t)+\gamma I_{k}(t)+\varepsilon Q_{k}(t)-\mu R_{k}(t)\right] d t-\sigma_{1} R_{k}(t) d B_{1}(t)
\end{aligned}\right.
$$

where $B_{i}(t)(t \geq 0)(i=1,2)$ are independent standard Brownian motions, $\sigma_{i}^{2}>0$, $(i=1,2)$ are the intensity of the white noise. 


\section{The existence of equilibrium of deterministic model}

In order to study the stochastic model (2) better, it is significant to study the deterministic model (1). Next, we discuss the existence of equilibrium and present the formula of the basic reproduction number.

Theorem 3.1 For model (1), define $R_{0}=\frac{\left\langle k^{2}\right\rangle}{\langle k\rangle} \frac{\beta \mu}{(\mu+\alpha)(v+\gamma+\lambda+\mu)}$, then the following propositions hold:

1. There always exists a disease-free equilibrium $E^{0}=\left\{\frac{\mu}{\mu+\alpha}, 0,0, \frac{\alpha}{\mu+\alpha}\right\}_{k=1}^{n}$.

2. There is an unique endemic equilibrium $E^{*}=\left\{S_{k}^{*}, I_{k}^{*}, Q_{k}^{*}, R_{k}^{*}\right\}_{k=1}^{n}$ if $R_{0}>1$, where

$$
\begin{gathered}
S_{k}^{*}=\frac{\mu\left[1+b\left(\Theta^{*}\right)^{2}\right]}{(\alpha+\mu)\left[1+b\left(\Theta^{*}\right)^{2}\right]+\beta k \Theta^{*}} ; \\
I_{k}^{*}=\frac{\mu \beta k \Theta^{*}}{(\nu+\gamma+\mu+\lambda)\left\{(\alpha+\mu)\left[1+b\left(\Theta^{*}\right)^{2}\right]+\beta k \Theta^{*}\right\}} ; \\
Q_{k}^{*}=\frac{\mu \nu \beta k \Theta^{*}}{(\nu+\gamma+\mu+\lambda)(\mu+\varepsilon+\lambda)\left\{(\alpha+\mu)\left[1+b\left(\Theta^{*}\right)^{2}\right]+\beta k \Theta^{*}\right\}} ; \\
R_{k}^{*}=\frac{\alpha(v+\gamma+\mu+\lambda)(\mu+\varepsilon+\lambda)\left[1+b\left(\Theta^{*}\right)^{2}\right]+[\varepsilon v+\gamma(\mu+\varepsilon+\lambda)] \beta k \Theta^{*}}{(\nu+\gamma+\mu+\lambda)(\mu+\varepsilon+\lambda)\left\{(\alpha+\mu)\left[1+b\left(\Theta^{*}\right)^{2}\right]+\beta k \Theta^{*}\right\}} .
\end{gathered}
$$

Proof One can obviously discover that $E^{0}$ is always an equilibrium of model (1). in stable state, the equilibrium $E^{*}=\left\{S_{k}^{*}, I_{k}^{*}, Q_{k}^{*}, R_{k}^{*}\right\}_{k=1}^{n}$ satisfy

$$
\left\{\begin{array}{l}
-\beta k S_{k}^{*} \frac{\Theta^{*}}{1+\mathrm{b}\left(\Theta^{*}\right)^{2}}-\alpha S_{k}^{*}-\mu S_{k}^{*}+\mu=0 \\
\beta k S_{k}^{*} \frac{\Theta^{*}}{1+\mathrm{b}\left(\Theta^{*}\right)^{2}}-\nu I_{k}^{*}-\gamma I_{k}^{*}-\mu I_{k}^{*}-\lambda I_{k}^{*}=0 \\
\nu I_{k}^{*}-\varepsilon Q_{k}^{*}-\mu Q_{k}^{*}-\lambda Q_{k}^{*}=0 \\
\alpha S_{k}^{*}+\gamma I_{k}^{*}+\varepsilon Q_{k}^{*}-\mu R_{k}^{*}=0
\end{array}\right.
$$

By calculation, we have 


$$
\left\{\begin{array}{l}
S_{k}^{*}=\frac{\mu\left[1+b\left(\Theta^{*}\right)^{2}\right]}{(\alpha+\mu)\left[1+b\left(\Theta^{*}\right)^{2}\right]+\beta k \Theta^{*}} ; \\
I_{k}^{*}=\frac{\mu \beta k \Theta^{*}}{(v+\gamma+\mu+\lambda)\left\{(\alpha+\mu)\left[1+b\left(\Theta^{*}\right)^{2}\right]+\beta k \Theta^{*}\right\}} ; \\
Q_{k}^{*}=\frac{\mu \nu \beta k \Theta^{*}}{(v+\gamma+\mu+\lambda)(\mu+\varepsilon+\lambda)\left\{(\alpha+\mu)\left[1+b\left(\Theta^{*}\right)^{2}\right]+\beta k \Theta^{*}\right\}} ; \\
R_{k}^{*}=\frac{\alpha(v+\gamma+\mu+\lambda)(\mu+\varepsilon+\lambda)\left[1+b\left(\Theta^{*}\right)^{2}\right]+[\varepsilon v+\gamma(\mu+\varepsilon+\lambda)] \beta k \Theta^{*}}{(v+\gamma+\mu+\lambda)(\mu+\varepsilon+\lambda)\left\{(\alpha+\mu)\left[1+b\left(\Theta^{*}\right)^{2}\right]+\beta k \Theta^{*}\right\}} .
\end{array}\right.
$$

Substituting $I_{k}^{*}$ into $\Theta$ yields

$$
\begin{aligned}
\Theta^{*} & =\frac{\sum_{k=1}^{n} k P(k)}{\langle k\rangle} \frac{\mu \beta k \Theta^{*}}{(v+\gamma+\mu+\lambda)\left\{(\alpha+\mu)\left[1+b\left(\Theta^{*}\right)^{2}\right]+\beta k \Theta^{*}\right\}} \\
& \triangleq g\left(\Theta^{*}\right) .
\end{aligned}
$$

Obviously, $\Theta^{*}=0$ is a trivial solution of Eq. (3), which corresponds to the diseasefree equilibrium of model (1). In order to make Eq. (3) has a nontrivial solution, the following conditions must be satisfied: $\left.\frac{d g\left(\Theta^{*}\right)}{d \Theta^{*}}\right|_{\Theta^{*}=0}>1$ and $g(1) \leq 1$.

$$
\text { i.e. }\left\{\begin{array}{l}
\frac{\sum_{k=1}^{n} k P(k)}{\langle k\rangle} \frac{\beta k \mu}{(\mu+\alpha)(v+\gamma+\mu+\lambda)}>1, \\
\frac{\sum_{k=1}^{n} k P(k)}{\langle k\rangle} \frac{\mu \beta k(\mu+\varepsilon)}{\beta k[v+(\mu+\varepsilon)(v+\gamma+\mu+\lambda)]+(\mu+\varepsilon)(\mu+\alpha)(\nu+\gamma+\mu+\lambda)(1+b)} \leq 1 .
\end{array}\right.
$$

In order for Eq. (4) to be true, we have to satisfy the inequality $\frac{\left\langle k^{2}\right\rangle}{\langle k\rangle} \frac{\beta \mu}{(\mu+\alpha)(v+\gamma+\mu+\lambda)}>$ 1 , define $R_{0}=\frac{\left\langle k^{2}\right\rangle}{\langle k\rangle} \frac{\beta \mu}{(\mu+\alpha)(v+\gamma+\mu+\lambda)}$, a nontrivial solution exists if and only if $R_{0}>1$.

Remark 3.1 $R_{0}=\frac{\left\langle k^{2}\right\rangle}{\langle k\rangle} \frac{\beta \mu}{(\mu+\alpha)(v+\gamma+\lambda+\mu)}$ is called the basic reproduction number of model (1), which is an important parameter to characterize the spread of infectious diseases, it represents the average number of secondary infectious infected by an individual of infective during whose whole course of disease in a group that all people are susceptible $[23,27]$. The basic reproduction number determines whether the disease will eventually break out. We can explain the formula for $R_{0}$ as follows, for the primary case, the average period of infection is $\frac{1}{v+\gamma+\lambda+\mu}$, during this period, the average number of susceptible infected by the primary case is $\beta \frac{\left\langle k^{2}\right\rangle}{\langle k\rangle} \frac{\mu}{\mu+\alpha}$. Accordingly, the basic reproduction number $R_{0}$ has biological significance.

Remark 3.2 The basic reproduction number $R_{0}$ means that epidemic threshold depends on the level of heterogeneity of the network $\left(\left\langle k^{2}\right\rangle /\langle k\rangle\right)$, transmission rate $(\beta)$, cure rate 
$(\gamma)$, mortality from diseases $(\lambda)$ and quarantine rate $(\nu)$, then if the heterogeneity of network and transmission rate increase, the risk of diseases outbreak rises, if the quarantine rate, cure rate, and mortality from diseases increase, the corresponding basic reproduction number $R_{0}$ and the final infection size $I_{k}^{*}$ decrease, that is, the risk of diseases outbreak decreases. In addition, the basic reproduction number is not dependent on the parameter $b$ of the non-monotone incidence rate, which consists with [19], but the final infection size of the diseases will decrease with the increases of parameter $b$.

\section{Preliminaries and necessary lemmas}

To begin with, some lemmas that will be used later are provided:

Definition 4.1 (Diffusion Matrix [28]) Let $X(t)$ be a homogeneous Markov process in $E_{d}$, and be described by the following stochastic differential equation

$$
d X(t)=b(X) d t+\sum_{r=1}^{k} g_{r}(X) d B_{r}(t)
$$

The diffusion matrix is defined as follows

$$
\mathbf{A}(x)=\left(a_{i j}(x)\right), a_{i j}(x)=\sum_{r=1}^{k} g_{r}^{i}(x) g_{r}^{j}(x) .
$$

Lemma 4.2 (ItÔ formula [29]) Let $x(t)$ be a d-dimensional It $\hat{O}$ process on $t \geq 0$ with the stochastic differential.

$$
d x(t)=f(t) d t+g(t) d B(t)
$$

where $f \in \mathcal{L}^{1}\left(\mathbb{R}_{+} ; \mathbb{R}^{d}\right)$ and $g \in \mathcal{L}^{2}\left(\mathbb{R}_{+} ; \mathbb{R}^{d \times m}\right)$. Let $V \in C^{2,1}\left(\mathbb{R}^{d} \times \mathbb{R}_{+} ; \mathbb{R}\right)$, then $V(x(t), t)$ is again an ItÔ process with the stochastic differential given by

$$
\begin{aligned}
d V(x(t), t) & =\left[V_{t}(x(t), t)+V_{x}(x(t), t) f(t)+\frac{1}{2} \operatorname{trace}\left(g^{T}(t) V_{x x}(x(t), t) g(t)\right)\right] d t \\
& +V_{x}(x(t), t) g(t) d B(t), \text { a.s. }
\end{aligned}
$$

Let differential operator $\mathcal{L}$ acts on a function $V \in C^{2,1}\left(\mathbb{R}^{d} \times\left[t_{0},+\infty\right] ; \mathbb{R}\right)$. Namely,

$$
\mathcal{L} V(x(t), t)=V_{t}(x(t), t)+V_{x}(x(t), t) f(t)+\frac{1}{2} \operatorname{trace}\left(g^{T}(t) V_{x x}(x(t), t) g(t)\right)
$$


Combining (5), we have

$$
d V(x(t), t)=\mathcal{L} V(x(t), t) d t+V_{x}(x(t), t) g(t) d B(t) a . s .
$$

Lemma 4.3 (Ergodic stationary distribution [28]) The Markov process $X(t)$ has a unique ergodic stationary distribution $\pi(\cdot)$, if there exists a bounded domain $D \subset E_{d}$ with regular boundary $\Gamma$ and.

$A_{1}$ : there is a positive number $M$ such that $\sum_{i, j=1}^{d} a_{i j}(x) \xi_{i} \xi_{j} \geq M|\xi|^{2}, x \in D$, $\xi \in \mathbb{R}^{d}$.

$A_{2}$ : there exists a nonnegative $C^{2}$-function $V$ such that $\mathcal{L} V$ is negative for any $E_{d} \backslash D$. then

$$
P_{x}\left\{\lim _{T \rightarrow \infty} \frac{1}{T} \int_{0}^{T} f(X(t)) d t=\int_{E_{d}} f(x) \pi(d x)\right\}=1 .
$$

for all $x \in E_{d}$, where $f(\cdot)$ is a integrable function with respect to the measure $\pi$.

Lemma 4.4 [30] If $a>0, b>0$ and $\frac{d x}{d t} \geq b-a x$, when $t \geq 0$ and $x(0) \geq 0$, we have $\lim _{t \rightarrow+\infty} \inf x(t) \geq \frac{b}{a}$.

If $a>0, b>0$ and $\frac{d x}{d t} \leq b-a x$, when $t \geq 0$ and $x(0) \geq 0$, we have $\lim _{t \rightarrow+\infty} \sup x(t) \leq \frac{b}{a}$.

\section{Existence and uniqueness of the positive solution}

In the rest of the paper, there is such a premise. Let $\left(\Omega, \mathcal{F},\left\{\mathcal{F}_{t}\right\}_{t \geq 0}, P\right)$ be a complete probability space with a filtration $\left\{\mathcal{F}_{t}\right\}_{t \geq 0}$ satisfying the usual conditions (i.e., it is right continuous and $\mathcal{F}_{0}$ contains all P-null sets), $B_{i}(t)(i=1,2)$ are defined on the complete probability space. Define

$$
\mathbb{R}_{+}^{4 n}=\left\{\left(x_{1}, x_{2}, \cdots, x_{4 n}\right) \mid x_{i} \geq 0, i=1,2, \cdots, 4 n\right\} .
$$

In order to have a better understanding stochastic epidemic model (2), it is of great significance to study the existence and uniqueness of the global positive solution.

Theorem 5.1 For any initial value $\left(S_{1}(0), \quad I_{1}(0), Q_{1}(0), \quad R_{1}(0), \cdots\right.$, $\left.S_{n}(0), \quad I_{n}(0), \quad Q_{n}(0), \quad R_{n}(0)\right) \in \mathbb{R}_{+}^{4 n}$, there is a unique solution $\left(S_{1}(t), I_{1}(t), Q_{1}(t), R_{1}(t), \cdots, S_{n}(t), I_{n}(t), Q_{n}(t), R_{n}(t)\right) \in \mathbb{R}_{+}^{4 n}$ of model (2) on $t \geq 0$, and the solution will remain in $\mathbb{R}_{+}^{4 n}$ with probability 1 .

Proof The coefficient of model (2) satisfy the local Lipschitz condition for any initial value $\left(S_{1}(0), I_{1}(0), Q_{1}(0), R_{1}(0), \cdots, S_{n}(0), I_{n}(0), Q_{n}(0)\right.$, $R_{n}(0) \quad \in \quad \mathbb{R}_{+}^{4 n}$, model (2) has a unique local solution $\left(S_{1}(t), I_{1}(t), Q_{1}(t), R_{1}(t), \cdots, S_{n}(t), I_{n}(t), Q_{n}(t), R_{n}(t)\right) \in \mathbb{R}_{+}^{4 n}$ on $t \in\left[0, \tau_{e}\right)$, where $\tau_{e}$ is the explosion time [29]. To manifest this solution is global, we only need 
to prove that $\tau_{e}=\infty$, a.s. let $d_{0}>0$ be arbitrarily large such that initial values lying with the interval $\left[1 / d_{0}, d_{0}\right]$. For each integer $d \geq d_{0}$, define the stopping time as follows:

$$
\begin{aligned}
& \tau_{d}=\inf \left\{t \in\left[0, \tau_{e}\right): \min \left\{S_{k}(t), I_{k}(t), Q_{k}(t), R_{k}(t)\right\} \leq \frac{1}{d}\right. \\
& \left.\cup \max \left\{S_{k}(t), I_{k}(t), Q_{k}(t), R_{k}(t)\right\} \geq d, k=1,2, \cdots, n\right\} .
\end{aligned}
$$

Set $\inf \emptyset=\infty$, one can easily see that $\tau_{d}$ is increasing as $d \rightarrow \infty$. Let $\tau_{\infty}=$ $\lim _{d \rightarrow \infty} \tau_{d}$, then $\tau_{\infty} \leq \tau_{e}$, a.s. accordingly, we only need to manifest $\tau_{\infty}=\infty$, a.s. if this proposition would not true, there is a constant $T>0$ and $\varepsilon \in(0,1)$ such that $P\left(\tau_{\infty} \leq T\right)>\varepsilon$. So there exists an integer $d_{1} \geq d_{0}$ such that $P\left(\tau_{d} \leq T\right) \geq \varepsilon$ for all $d \geq d_{1}$.

Define $C^{2}$-function $V: \mathbb{R}_{+}^{4 n} \rightarrow \mathbb{R}_{+}$by

$$
\begin{aligned}
& V(\left.S_{1}, I_{1}, Q_{1}, R_{1}, \cdots, S_{n}, I_{n}, Q_{n}, R_{n}\right) \\
&= \sum_{k=1}^{n}\left[\left(S_{k}-h-h \ln \frac{S_{k}}{h}\right)+\left(I_{k}-1-\ln I_{k}\right)+\left(Q_{k}-1-\ln Q_{k}\right)\right. \\
&\left.\quad+\left(R_{k}-1-\ln R_{k}\right)\right] .
\end{aligned}
$$

where $h$ is a positive constant satisfying

$$
h \beta n^{2}(n+1) \max _{1 \leq k \leq n} P(k)-2(\lambda+\mu)\langle k\rangle=0 .
$$

Using ItÔ formula, we derive

$$
\begin{aligned}
& d V=\mathcal{L} V d t-\sigma_{1} \sum_{k=1}^{n}\left[\left(S_{k}-h\right)+\left(I_{k}-1\right)+\left(Q_{k}-1\right)+\left(R_{k}-1\right)\right] d B_{1}(t) \\
& -\sigma_{2} \sum_{k=1}^{n}\left[\left(I_{k}-1\right)+\left(Q_{k}-1\right)\right] d B_{2}(t) .
\end{aligned}
$$

where

$$
\begin{aligned}
\mathcal{L} V= & \sum_{k=1}^{n}\left\{\left(1-\frac{h}{S_{k}}\right)\left[-\beta k S_{k} \frac{\Theta}{1+b \Theta^{2}}-\alpha S_{k}-\mu S_{k}+\mu\right]+\frac{h \sigma_{1}^{2}}{2}\right\} \\
& +\sum_{k=1}^{n}\left\{\left(1-\frac{1}{I_{k}}\right)\left[\beta k S_{k} \frac{\Theta}{1+b \Theta^{2}}-\nu I_{k}-\gamma I_{k}-\lambda I_{k}-\mu I_{k}\right]+\frac{\sigma_{1}^{2}+\sigma_{2}^{2}}{2}\right\} \\
& +\sum_{k=1}^{n}\left\{\left(1-\frac{1}{Q_{k}}\right)\left[v I_{k}-\varepsilon Q_{k}-\lambda Q_{k}-\mu Q_{k}\right]+\frac{\sigma_{1}^{2}+\sigma_{2}^{2}}{2}\right\} \\
& +\sum_{k=1}^{n}\left\{\left(1-\frac{1}{R_{k}}\right)\left[\alpha S_{k}+\gamma I_{k}+\varepsilon Q_{k}-\mu R_{k}\right]+\frac{\sigma_{1}^{2}}{2}\right\}
\end{aligned}
$$




$$
\begin{aligned}
& =\sum_{k=1}^{n}\left[\mu-\mu\left(S_{k}+I_{k}+Q_{k}+R_{k}\right)+\beta k \frac{h \Theta}{1+b \Theta^{2}}+h(\alpha+\mu)\right. \\
& -\frac{h \mu}{S_{k}}-\beta k \frac{S_{k}}{I_{k}} \frac{\Theta}{1+b \Theta^{2}}+v+\gamma+\mu+\lambda-v \frac{I_{k}}{Q_{k}}-\lambda I_{k}+\varepsilon \\
& \left.+\mu+\lambda-\alpha \frac{S_{k}}{R_{k}}-\gamma \frac{I_{k}}{R_{k}}-\varepsilon \frac{Q_{k}}{R_{k}}-\lambda Q_{k}+\mu+\frac{(1+h) \sigma_{1}^{2}}{2}+\sigma_{1}^{2}+\sigma_{2}^{2}\right] \\
& \leq \sum_{k=1}^{n}\left[\beta k \frac{h \Theta}{1+b \Theta^{2}}+h(\alpha+\mu)+v+\gamma+\varepsilon+2 \lambda+4 \mu-(\lambda+\mu) I_{k}+\frac{(1+h) \sigma_{1}^{2}}{2}+\sigma_{1}^{2}+\sigma_{2}^{2}\right] .
\end{aligned}
$$

Notice that

$$
\begin{aligned}
& \sum_{k=1}^{n}\left[\beta k \frac{h \Theta}{1+b \Theta^{2}}-(\lambda+\mu) I_{k}\right] \leq \sum_{k=1}^{n}\left[\beta k h \Theta-(\lambda+\mu) I_{k}\right] \\
& \leq \sum_{k=1}^{n}\left[\frac{\beta h n^{2}(n+1) \max _{1 \leq k \leq n} P(k)}{2\langle k\rangle}-(\lambda+\mu)\right] I_{k}=0 .
\end{aligned}
$$

Thus

$$
\mathcal{L} V \leq \sum_{k=1}^{n}\left[h(\alpha+\mu)+\gamma+\varepsilon+2 \lambda+\nu+4 \mu+\frac{(1+h) \sigma_{1}^{2}}{2}+\sigma_{1}^{2}+\sigma_{2}^{2}\right]=: H
$$

Accordingly

$$
\begin{aligned}
& d V \leq H-\sigma_{1} \sum_{k=1}^{n}\left[\left(S_{k}-h\right)+\left(I_{k}-1\right)+\left(Q_{k}-1\right)+\left(R_{k}-1\right)\right] d B_{1}(t) \\
& \quad-\sigma_{2} \sum_{k=1}^{n}\left[\left(I_{k}-1\right)+\left(Q_{k}-1\right)\right] d B_{2}(t) .
\end{aligned}
$$

By integrating both sides of Eq. (6) and taking the expected value as follows:

$$
\begin{aligned}
& E\left[V \left(S_{1}\left(\tau_{d} \wedge T\right), I_{1}\left(\tau_{d} \wedge T\right), Q_{1}\left(\tau_{d} \wedge T\right), R_{1}\left(\tau_{d} \wedge T\right), \cdots, S_{n}\left(\tau_{d} \wedge T\right)\right.\right. \\
& \left.\left.I_{n}\left(\tau_{d} \wedge T\right), Q_{n}\left(\tau_{d} \wedge T\right), R_{n}\left(\tau_{d} \wedge T\right)\right)\right] \\
& =V\left[S_{1}(0), I_{1}(0), Q_{1}(0), R_{1}(0), \cdots, S_{n}(0), I_{n}(0), Q_{n}(0), R_{n}(0)\right]+E\left(\int_{0}^{\tau_{d} \wedge T} \mathcal{L} V d t\right) \\
& \leq V\left[S_{1}(0), I_{1}(0), Q_{1}(0), R_{1}(0), \cdots, S_{n}(0), I_{n}(0), Q_{n}(0), R_{n}(0)\right]+H T .
\end{aligned}
$$

Let $\Omega_{d}=\left\{\tau_{d} \leq T\right\}$ for $d \geq d_{1}$, then $P\left(\Omega_{d}\right) \geq \varepsilon$. For any $\varphi \in \Omega_{d}$, there exists at least one of $S_{k}\left(\tau_{d}, \varphi\right), I_{k}\left(\tau_{d}, \varphi\right), Q_{k}\left(\tau_{d}, \varphi\right)$ and $R_{k}\left(\tau_{d}, \varphi\right)$ equaling $d$ or $\frac{1}{d}$. Consequently 


$$
\begin{aligned}
& V\left(S_{1}\left(\tau_{d}\right), I_{1}\left(\tau_{d}\right), Q_{1}\left(\tau_{d}\right), R_{1}\left(\tau_{d}\right), \cdots, S_{n}\left(\tau_{d}\right), I_{n}\left(\tau_{d}\right), Q_{n}\left(\tau_{d}\right), R_{n}\left(\tau_{d}\right)\right) \\
& \geq(d-1-\ln d) \wedge\left(\frac{1}{d}-1+\ln d\right) .
\end{aligned}
$$

One can easily derive from (7) and (8)

$$
\begin{aligned}
& V\left[S_{1}(0), I_{1}(0), Q_{1}(0), R_{1}(0), \ldots, S_{n}(0), I_{n}(0), Q_{n}(0), R_{n}(0)\right]+H T \\
& \geq E\left[V \left(S_{1}\left(\tau_{d} \wedge T\right), I_{1}\left(\tau_{d} \wedge T\right), Q_{1}\left(\tau_{d} \wedge T\right), R_{1}\left(\tau_{d} \wedge T\right), \ldots, S_{n}\left(\tau_{d} \wedge T\right),\right.\right. \\
& \left.\left.I_{n}\left(\tau_{d} \wedge T\right), Q_{n}\left(\tau_{d} \wedge T\right), R_{n}\left(\tau_{d} \wedge T\right)\right)\right] \\
& =E\left[1_{\Omega_{d(\varphi)}} S_{1}\left(\tau_{d}, \varphi\right), I_{1}\left(\tau_{d}, \varphi\right), Q_{1}\left(\tau_{d}, \varphi\right), R_{1}\left(\tau_{d}, \varphi\right), \ldots, S_{n}\left(\tau_{d}, \varphi\right),\right. \\
& \left.I_{n}\left(\tau_{d}, \varphi\right), Q_{n}\left(\tau_{d}, \varphi\right), R_{n}\left(\tau_{d}, \varphi\right)\right] \\
& \geq \varepsilon\left[(d-1-\ln d) \wedge\left(\frac{1}{d}-1+\ln d\right)\right] .
\end{aligned}
$$

here $1_{\Omega_{d(\varphi)}}$ is the indicator function of $\Omega_{d}$. When $d \rightarrow \infty$, we have

$\infty>V\left[S_{1}(0), I_{1}(0), Q_{1}(0), R_{1}(0), \cdots, S_{n}(0), I_{n}(0), Q_{n}(0), R_{n}(0)\right]+H T \geq \infty$.

This leads a contradiction, accordingly $\tau_{\infty}=\infty$, a.s. The proof is completed.

Remark 5.1 According to Theorem 5.1, one can easily obtained that for any initial value $\left(S_{k}(0), I_{k}(0), Q_{k}(0), R_{k}(0)\right)_{k=1}^{n} \in \mathbb{R}_{+}^{4 n}$, the stochastic model (2) has a unique solution $\left(S_{k}(t), I_{k}(t), Q_{k}(t), R_{k}(t)\right)_{k=1}^{n} \in \mathbb{R}_{+}^{4 n}$ on $t \geq 0$, which is almost surely.

Lemma 5.1 For any initial values $\left(S_{k}(0), I_{k}(0), Q_{k}(0), R_{k}(0)\right)_{k=1}^{n} \in \mathbb{R}_{+}^{4 n}$ and $N_{k}(0)=S_{k}(0)+I_{k}(0)+Q_{k}(0)+R_{k}(0)=1,(k=1,2, \cdots n)$, the solution of stochastic model (2)has the property that $N_{k}(t)=S_{k}(t)+I_{k}(t)+Q_{k}(t)+R_{k}(t)=1,(t \geq 0)$.

Proof From (1), we obtain.

$$
\frac{d N_{k}(t)}{d t}=\mu-\mu N_{k}(t)-\lambda\left(I_{k}(t)+Q_{k}(t)\right) \leq \mu-\mu N_{k}(t) .
$$

thus

$$
N_{k}(t) \leq N_{k}(0) e^{-\mu t}+1
$$

therefore

$$
\lim _{t \rightarrow+\infty} \sup N_{k}(t)=1
$$

Accordingly, the positively invariant set of stochastic model (2) is

$$
\Gamma=\left\{\left(S_{1}, I_{1}, Q_{1}, R_{1}, \ldots, S_{n}, I_{n}, Q_{n}, R_{n}\right)\right.
$$




$$
\left.\in \mathbb{R}_{+}^{4 n} \mid S_{k}(t)+I_{k}(t)+Q_{k}(t)+R_{k}(t)=1, \quad k=1,2, \ldots, n\right\}
$$

Next, we always presume that $\left(S_{1}(t), I_{1}(t), Q_{1}(t), R_{1}(t), \cdots, S_{n}(t), I_{n}(t), Q_{n}(t)\right.$, $\left.R_{n}(t)\right) \in \Gamma$.

\section{Extinction of the disease}

One of the most important problems in epidemiology is how to regulate the dynamics of diseases so that the diseases extinct in a long time, the conditions of diseases extinction is manifested by noise intensity and system parameters.

Theorem 6.1 Let $\left(S_{1}(t), I_{1}(t), Q_{1}(t), R_{1}(t), \cdots, S_{n}(t), I_{n}(t), Q_{n}(t), R_{n}(t)\right) \quad \in$ $\mathbb{R}_{+}^{4 n}$ be the solution of model (2) with any initial value $\left(S_{1}(0), I_{1}(0), Q_{1}(0), R_{1}(0), \cdots, S_{n}(0), I_{n}(0), Q_{n}(0), R_{n}(0)\right) \in \mathbb{R}_{+}^{4 n}$, if $R_{0}<1$, then

$$
\lim _{t \rightarrow \infty} \sup \frac{\ln I_{k}(t)}{t} \leq\left(R_{0}-1\right)-\frac{1}{2}\left(\sigma_{1}^{2}+\sigma_{2}^{2}\right)<0, \text { a.s. }
$$

i.e., $I_{k}(t)$ tends to zero exponentially almost surely.

Proof Based on model (2), we derive.

$$
\begin{aligned}
d \Theta & =\sum_{k=1}^{n} \frac{k P(k)}{\langle k\rangle}\left\{\left[\beta k S_{k} \frac{\Theta}{1+b \Theta^{2}}-\nu I_{k}-\gamma I_{k}-\lambda I_{k}-\mu I_{k}\right] d t-\sigma_{1} I_{k} d B_{1}(t)-\sigma_{2} I_{k} d B_{2}(t)\right\} \\
& =\frac{\Theta}{1+b \Theta^{2}}\left[\sum_{k=1}^{n} \frac{k P(k)}{\langle k\rangle} \beta k S_{k}-(\nu+\gamma+\lambda+\mu)\left(1+b \Theta^{2}\right)\right] d t \\
& -\sum_{k=1}^{n} \frac{k P(k)}{\langle k\rangle}\left(\sigma_{1} I_{k} d B_{1}(t)+\sigma_{2} I_{k} d B_{2}(t)\right) .
\end{aligned}
$$

Applying ItÔ formula to model (2), we obtain

$$
d(\ln \Theta)=\mathcal{L}(\ln \Theta) d t-\sum_{k=1}^{n} \frac{k P(k)}{\Theta\langle k\rangle}\left(\sigma_{1} I_{k} d B_{1}(t)+\sigma_{2} I_{k} d B_{2}(t)\right)
$$

where

$$
\begin{aligned}
\mathcal{L}(\ln \Theta) & =\frac{1}{1+b \Theta^{2}}\left[\sum_{k=1}^{n} \frac{k P(k)}{\langle k\rangle} \beta k S_{k}-(v+\gamma+\lambda+\mu)\left(1+b \Theta^{2}\right)\right]-\frac{1}{2 \Theta^{2}} \sum_{k=1}^{n}\left(\frac{k P(k)}{\langle k\rangle} \sigma_{1} I_{k}\right)^{2} \\
& -\frac{1}{2 \Theta^{2}} \sum_{k=1}^{n}\left(\frac{k P(k)}{\langle k\rangle} \sigma_{2} I_{k}\right)^{2} .
\end{aligned}
$$


By using the Cauchy Inequality: $\left(\sum_{k=1}^{n} a_{k} b_{k}\right)^{2} \leq \sum_{k=1}^{n}\left(a_{k}\right)^{2} \sum_{k=1}^{n}\left(b_{k}\right)^{2}$, we obtain

$$
\begin{aligned}
\Theta^{2} & =\left(\sum_{k=1}^{n} \frac{k P(k) I_{k}}{\langle k\rangle}\right)^{2} \\
& =\left(\sum_{k=1}^{n} \frac{k P(k) \sigma_{i} I_{k}}{\langle k\rangle} \frac{1}{\sigma_{i}}\right)^{2} \\
& =\sum_{k=1}^{n}\left(\frac{k P(k) \sigma_{i} I_{k}}{\langle k\rangle}\right)^{2} \sum_{k=1}^{n}\left(\frac{1}{\sigma_{i}}\right)^{2}(i=1,2) .
\end{aligned}
$$

Combining (9) with (10), we obtain

$$
\mathcal{L}(\ln \Theta) \leq \frac{1}{1+b \Theta^{2}}\left[\sum_{k=1}^{n} \frac{k P(k)}{\langle k\rangle} \beta k S_{k}-(\nu+\gamma+\mu+\lambda)\left(1+b \Theta^{2}\right)\right]-\frac{1}{2}\left(\frac{1}{\sigma_{1}^{2}}\right)^{-1}-\frac{1}{2}\left(\frac{1}{\sigma_{2}^{2}}\right)^{-1} .
$$

\section{Consequently}

$$
\begin{aligned}
d(\ln \Theta) & \leq \frac{1}{1+b \Theta^{2}}\left[\sum_{k=1}^{n} \frac{k P(k)}{\langle k\rangle} \beta k S_{k}-(v+\gamma+\lambda+\mu)\left(1+b \Theta^{2}\right)\right]-\frac{1}{2}\left(\frac{1}{\sigma_{1}^{2}}\right)^{-1} \\
& -\frac{1}{2}\left(\frac{1}{\sigma_{2}^{2}}\right)^{-1}-\sum_{k=1}^{n} \frac{k P(k)}{\Theta\langle k\rangle}\left(\sigma_{1} I_{k} d B_{1}(t)+\sigma_{2} I_{k} d B_{2}(t)\right) .
\end{aligned}
$$

Integrating both sides of (11) from 0 to $t$

$$
\begin{aligned}
& \ln \Theta(t)-\ln \Theta(0) \\
& \leq \int_{0}^{t} \frac{1}{1+b \Theta^{2}}\left[\sum_{k=1}^{n} \frac{k P(k)}{\langle k\rangle} \beta k S_{k}-(v+\gamma+\mu)\left(1+b \Theta^{2}\right)\right] d t-\int_{0}^{t} \frac{1}{2}\left(\frac{1}{\sigma_{1}^{2}}\right)^{-1} d t \\
& \quad-\int_{0}^{t} \frac{1}{2}\left(\frac{1}{\sigma_{2}^{2}}\right)^{-1} d t-\int_{0}^{t} \sum_{k=1}^{n} \frac{k P(k)}{\Theta\langle k\rangle}\left(\sigma_{1} I_{k} d B_{1}(s)+\sigma_{2} I_{k} d B_{2}(s)\right) .
\end{aligned}
$$

Let

$$
Z_{i}(t)=: \int_{0}^{t} \sum_{k=1}^{n} \frac{k P(k)}{\Theta\langle k\rangle} \sigma_{i} I_{k} d B_{i}(s)(i=1,2)
$$


which is local continuous martingale and $Z(0)=0$, moreover

$$
\begin{aligned}
& \frac{1}{t}\left\langle Z_{i}, Z_{i}\right\rangle_{t}=\frac{1}{t} \int_{0}^{t} \frac{1}{\Theta^{2}}\left(\sum_{k=1}^{n} \frac{k P(k)}{\langle k\rangle} \sigma_{i} I_{k}\right)^{2} d s \\
& \quad=\frac{1}{t} \int_{0}^{t}\left(\sum_{k=1}^{n} \frac{k P(k) I_{k}}{\langle k\rangle}\right)^{-2}\left(\sum_{k=1}^{n} \frac{k P(k)}{\langle k\rangle} \sigma_{i} I_{k}\right)^{2} d s \\
& \quad \leq \max _{1 \leq k \leq n}\left\{\sigma_{i}^{2}\right\}<\infty(i=1,2) .
\end{aligned}
$$

By Strong Law of Large Numbers [31], we derive

$$
\lim _{t \rightarrow \infty} \frac{Z_{i}(t)}{t}=0, \text { a.s. }
$$

Thus

$$
\begin{aligned}
\ln \Theta(t) & \leq \ln \Theta(0)+\int_{0}^{t} \frac{1}{1+b \Theta^{2}}\left[\sum_{k=1}^{n} \frac{k P(k)}{\langle k\rangle} \beta k S_{k}-(v+\gamma+\lambda+\mu)\left(1+b \Theta^{2}\right)\right] d t \\
& -\int_{0}^{t} \frac{1}{2}\left(\frac{1}{\sigma_{1}^{2}}\right)^{-1} d t-\int_{0}^{t} \frac{1}{2}\left(\frac{1}{\sigma_{2}^{2}}\right)^{-1} d t
\end{aligned}
$$

When $R_{0}<1, \lim _{t \rightarrow \infty} S_{k}(t)=\frac{\mu}{\mu+\alpha}$.

Accordingly

$$
\begin{aligned}
& \lim _{t \rightarrow \infty} \sup \frac{\ln \Theta(t)}{t} \\
& \leq \frac{1}{1+b \Theta^{2}}\left[\sum_{k=1}^{n} \frac{k P(k)}{\langle k\rangle} \frac{\mu}{\mu+\alpha} \beta k-(v+\gamma+\lambda+\mu)\left(1+b \Theta^{2}\right)\right]-\frac{1}{2}\left(\sigma_{1}^{2}+\sigma_{2}^{2}\right) \\
& \quad \leq \sum_{k=1}^{n} \frac{k P(k)}{\langle k\rangle} \frac{\mu \beta k}{\mu+\alpha}-(v+\gamma+\lambda+\mu)-\frac{1}{2}\left(\sigma_{1}^{2}+\sigma_{2}^{2}\right) \\
& =(v+\gamma+\lambda+\mu)\left(R_{0}-1\right)-\frac{1}{2}\left(\sigma_{1}^{2}+\sigma_{2}^{2}\right) \\
& \quad<0
\end{aligned}
$$

Remark 6.1 According to Theorem 6.1, one can easily find that $R_{0}$ exhibits behaviors: if $R_{0}<1, I_{k}(t)$ tends to zero exponentially almost surely, that is to say, the diseases extinct eventually. In particular, the diseases will die out eventually with probability one if $\sigma_{1}^{2}+\sigma_{2}^{2}$ enough large, regardless of whether $R_{0}$ is greater than one.

Remark 6.2 From Theorem 6.1, when $R_{0}<1$, the diseases will be extinct with probability 1 . From $R_{0}=\frac{\left\langle k^{2}\right\rangle}{\langle k\rangle} \frac{\beta \mu}{(\mu+\alpha)(v+\gamma+\lambda+\mu)}$, the basic reproduction number $R_{0}$ decreases 
with the increase of quarantine rate $v$. Therefore, quarantine measures accelerate the extinction of the diseases.

\section{Stationary distribution and ergodicity of stochastic model}

In epidemiology, people pay more attention to the diseases will prevail in the population. For deterministic models, we can solve this problem by demonstrating that the endemic equilibrium is globally asymptotically stable. However, it is impossible to find the endemic equilibrium of stochastic models, so we verify the existence and ergodicity of the stationary distribution of the stochastic model (2) in order to explore the prevail time of the diseases in the population.

Theorem 7.1 Let $\left(S_{1}(t), I_{1}(t), Q_{1}(t), R_{1}(t), \cdots, S_{n}(t), I_{n}(t), Q_{n}(t), R_{n}(t)\right) \quad \in$ $\mathbb{R}_{+}^{4 n}$ be the solution of model (2) with any initial value $\left(S_{1}(0), I_{1}(0), Q_{1}(0), R_{1}(0), \cdots, S_{n}(0), I_{n}(0), Q_{n}(0), R_{n}(0)\right) \quad \in \quad \mathbb{R}_{+}^{4 n}$, When $R_{0}>1$, if the conditions $\omega_{i}>0(i=1,2,3,4), F<$ $\min \left\{\omega_{1}\left(S_{k}^{*}\right)^{2}, \omega_{2}\left(I_{k}^{*}\right)^{2}, \omega_{3}\left(Q_{k}^{*}\right)^{2}, \omega_{4}\left(R_{k}^{*}\right)^{2}\right\}$ are satisfied, then model (2) has a unique stationary distribution and ergodic property. where.

$$
\begin{aligned}
\omega_{1}= & \sum_{k=1}^{n} \xi(k)\left(\alpha+\mu-\frac{\alpha}{2}-\sigma_{1}^{2}\right), \quad \omega_{2}=\sum_{k=1}^{n} \xi(k)\left(\frac{v}{2}+\frac{\gamma}{2}+\mu+\lambda-\sigma_{1}^{2}-\sigma_{2}^{2}\right), \\
\omega_{3}= & \sum_{k=1}^{n} \xi(k)\left(\frac{\varepsilon}{2}+\mu+\lambda-\frac{v}{2}-\sigma_{1}^{2}-\sigma_{2}^{2}\right), \quad \omega_{4}=\sum_{k=1}^{n} \xi(k)\left(\mu-\frac{\alpha}{2}-\frac{\gamma}{2}-\frac{\varepsilon}{2}-\sigma_{1}^{2}\right), \\
F= & \sum_{k=1}^{n} \xi(k)\left[l_{1}\left(S_{k}^{*}\right)^{2}+l_{2}\left(I_{k}^{*}\right)^{2}+l_{2}\left(Q_{k}^{*}\right)^{2}+l_{1}\left(R_{k}^{*}\right)^{2}\right. \\
& \left.+\frac{1}{2} \frac{(\alpha+2 \mu+v+\gamma+\lambda)\left(1+b\left(\Theta^{*}\right)^{2}\right)\langle k\rangle}{\beta\left\langle k^{2}\right\rangle}\left(\sigma_{1}^{2}+\sigma_{2}^{2}\right) I_{k}^{*}\right] \\
l_{1}= & \sigma_{1}^{2}, \quad l_{2}=\sigma_{1}^{2}+\sigma_{2}^{2}, \quad \xi(k)=\frac{k P(k)}{\langle k\rangle} .
\end{aligned}
$$

Proof One can easily obtained that for any initial value $\left(S_{1}(0), I_{1}(0), Q_{1}(0), R_{1}(0), \cdots, S_{n}(0), \quad I_{n}(0), Q_{n}(0), R_{n}(0)\right) \quad \in \quad \mathbb{R}_{+}^{4 n}$, there is a unique global solution $\left(S_{1}(t), I_{1}(t), Q_{1}(t), R_{1}(t), \cdots, S_{n}(t), \quad I_{n}(t)\right.$, $\left.Q_{n}(t), R_{n}(t)\right) \in \mathbb{R}_{+}^{4 n}$ by Theorem 5.1.

The diffusion matrix of model (2) is shown by 


$$
\begin{aligned}
\mathbf{A}(x) & =\left(a_{i j}(x)\right)_{4 n \times 4 n} \\
= & \left\{\sigma_{1}^{2} S_{1}^{2}, \ldots, \sigma_{1}^{2} S_{n}^{2},\left(\sigma_{1}^{2}+\sigma_{2}^{2}\right) I_{1}^{2}, \ldots,\left(\sigma_{1}^{2}+\sigma_{2}^{2}\right) I_{n}^{2},\left(\sigma_{1}^{2}+\sigma_{2}^{2}\right) Q_{1}^{2},\right. \\
& \left.\ldots\left(\sigma_{1}^{2}+\sigma_{2}^{2}\right) Q_{n}^{2}, \sigma_{1}^{2} R_{1}^{2}, \ldots, \sigma_{1}^{2} R_{n}^{2}\right\} .
\end{aligned}
$$

where $x=\left(S_{1}, \cdots, S_{n}, I_{1}, \cdots, I_{n}, Q_{1}, \cdots, Q_{n}, R_{1}, \cdots, R_{n}\right)$.

Choosing $C=\min \left\{\sigma_{1}^{2} S_{k}^{2}, \quad\left(\sigma_{1}^{2}+\sigma_{2}^{2}\right) I_{k}^{2}, \quad\left(\sigma_{1}^{2}+\sigma_{2}^{2}\right) Q_{k}^{2}\right.$, $\left.\sigma_{1}^{2} R_{k}^{2} \mid\left(S_{k}, I_{k}, Q_{k}, R_{k}\right) \in \bar{D}_{\sigma}, k=1,2, \cdots, n\right\} \quad>\quad 0$. for any $\left(S_{1}, \ldots, S_{n}, I_{1}, \ldots, I_{n}, Q_{1}, \ldots, Q_{n}, R_{1}, \ldots, R_{n} \in \bar{D}_{\sigma}\right)$ and $\xi=\left(\xi_{1}, \cdots, \xi_{4 n}\right) \in$ $\mathbb{R}_{+}^{4 n}$.

one can obtain that

$$
\begin{aligned}
& \sum_{i, j=1}^{4 n+1} a_{i j} \xi_{i} \xi_{j}=\sigma_{1}^{2} S_{1}^{2} \xi_{1}^{2}+\cdots+\sigma_{1}^{2} S_{n}^{2} \xi_{n}^{2}+\left(\sigma_{1}^{2}+\sigma_{2}^{2}\right) I_{1}^{2} \xi_{n+1}^{2}+\cdots+\left(\sigma_{1}^{2}+\sigma_{2}^{2}\right) I_{n}^{2} \xi_{2 n}^{2} \\
& \quad\left(\sigma_{1}^{2}+\sigma_{2}^{2}\right) Q_{1}^{2} \xi_{2 n+1}^{2}+\cdots+\left(\sigma_{1}^{2}+\sigma_{2}^{2}\right) Q_{n}^{2} \xi_{3 n}^{2}+\sigma_{1}^{2} R_{1}^{2} \xi_{3 n+1}^{2}+\cdots+\sigma_{1}^{2} R_{n}^{2} \xi_{4 n}^{2} \\
& \quad \geq C|\xi|^{2}
\end{aligned}
$$

where $|\xi|=\sqrt{\sum_{i=1}^{4 n} \xi_{i}^{2}}$, this manifests that condition $A_{1}$ of Lemma 4.3 is satisfied.

To demonstrate that condition $A_{2}$ of Lemma 4.3 is satisfied, we further define the Lyapunov functions as follows:

$$
\begin{aligned}
& V\left(S_{1}, I_{1}, Q_{1}, R_{1}, \cdots S_{n}, I_{n}, Q_{n}, R_{n}\right) \\
& =\frac{1}{2} \sum_{k=1}^{n} \xi(k)\left(S_{k}-S_{k}^{*}+I_{k}-I_{k}^{*}\right)^{2}+\eta^{*}\left(\Theta-\Theta^{*}-\Theta^{*} \ln \frac{\Theta}{\Theta^{*}}\right)+\frac{1}{2} \sum_{k=1}^{n} \xi(k)\left(Q_{k}-Q_{k}^{*}\right)^{2} \\
& \quad+\frac{1}{2} \sum_{k=1}^{n} \xi(k)\left(R_{k}-R_{k}^{*}\right)^{2} \\
& =: V_{1 k}+\eta^{*} V_{2 k}+V_{3 k}+V_{4 k} .
\end{aligned}
$$

where $\eta^{*}=\frac{(\alpha+2 \mu+\nu+\gamma+\lambda)\left(1+b\left(\Theta^{*}\right)^{2}\right)\langle k\rangle}{\beta\left\langle k^{2}\right\rangle}$.

By applying ItÔ formula and $a^{2}+b^{2} \geq 2 a b$, we first obtain

$$
\begin{aligned}
& d V_{1 k}=\sum_{k=1}^{n} \xi(k)\left(S_{k}-S_{k}^{*}+I_{k}-I_{k}^{*}\right)\left(\mu-\alpha S_{k}-\mu S_{k}-\nu I_{k}-\gamma I_{k}-\mu I_{k}-\lambda I_{k}-\sigma_{1} S_{k} d B_{1}\right. \\
& \left.\quad-\sigma_{1} I_{k} d B_{1}-\sigma_{2} I_{k} d B_{2}\right)+\frac{1}{2} \sum_{k=1}^{n} \xi(k) \sigma_{1}^{2} S_{k}^{2}+\frac{1}{2} \sum_{k=1}^{n} \xi(k) \sigma_{1}^{2} I_{k}^{2}+\frac{1}{2} \sum_{k=1}^{n} \xi(k) \sigma_{2}^{2} I_{k}^{2} \\
& =\mathcal{L} V_{1 k} d t-\sum_{k=1}^{n} \xi(k)\left(S_{k}-S_{k}^{*}+I_{k}-I_{k}^{*}\right)\left(\sigma_{1} S_{k} d B_{1}+\sigma_{1} I_{k} d B_{1}+\sigma_{2} I_{k} d B_{2}\right)
\end{aligned}
$$


Thus

$$
\begin{aligned}
\mathcal{L} V_{1 k}= & \sum_{k=1}^{n} \xi(k)\left(S_{k}-S_{k}^{*}+I_{k}-I_{k}^{*}\right)\left(\mu-\alpha S_{k}-\mu S_{k}-v I_{k}-\gamma I_{k}-\mu I_{k}-\lambda I_{k}\right) \\
& +\frac{1}{2} \sum_{k=1}^{n} \xi(k) \sigma_{1}^{2} S_{k}^{2}+\frac{1}{2} \sum_{k=1}^{n} \xi(k) \sigma_{1}^{2} I_{k}^{2}+\frac{1}{2} \sum_{k=1}^{n} \xi(k) \sigma_{2}^{2} I_{k}^{2} \\
& \leq \sum_{k=1}^{n} \xi(k)\left[-\left(\alpha+\mu-\sigma_{1}^{2}\right)\left(S_{k}-S_{k}^{*}\right)^{2}-\left(v+\gamma+\mu+\lambda-\sigma_{1}^{2}-\sigma_{2}^{2}\right)\left(I_{k}-I_{k}^{*}\right)^{2}\right. \\
& \left.-(\alpha+2 \mu+v+\gamma+\lambda)+\left(S_{k}-S_{k}^{*}\right)\left(I_{k}-I_{k}^{*}\right)\right]+\sum_{k=1}^{n} \xi(k)\left[\sigma_{1}^{2}\left(S_{k}^{*}\right)^{2}+\sigma_{1}^{2}\left(I_{k}^{*}\right)^{2}+\sigma_{2}^{2}\left(I_{k}^{*}\right)^{2}\right] \\
& =-\left(\alpha+\mu-\sigma_{1}^{2}\right) \sum_{k=1}^{n} \xi(k)\left(S_{k}-S_{k}^{*}\right)^{2}-\left(v+\gamma+\mu+\lambda-\sigma_{1}^{2}-\sigma_{2}^{2}\right) \sum_{k=1}^{n} \xi(k)\left(I_{k}-I_{k}^{*}\right)^{2}- \\
& (\alpha+2 \mu+v+\gamma+\lambda)\left(S_{k}-S_{k}^{*}\right)\left(\Theta-\Theta^{*}\right) \\
& +\sum_{k=1}^{n} \xi(k)\left[\sigma_{1}^{2}\left(S_{k}^{*}\right)^{2}+\sigma_{1}^{2}\left(I_{k}^{*}\right)^{2}+\sigma_{2}^{2}\left(I_{k}^{*}\right)^{2}\right] .
\end{aligned}
$$

Second, we derive

$$
\begin{aligned}
d V_{2 k}= & \left(1-\frac{\Theta^{*}}{\Theta}\right) \sum_{k=1}^{n} \frac{k P(k)}{\langle k\rangle}\left[\left(\beta k S_{k} \frac{\Theta}{1+b \Theta^{2}}-v I_{k}-\gamma I_{k}-\mu I_{k}-\lambda I_{k}\right) d t-\sigma_{1} I_{k} d B_{1}-\sigma_{2} I_{k} d B_{2}\right] \\
& +\frac{1}{2} \sum_{k=1}^{n} \frac{\Theta^{*}}{\Theta^{2}}\left(\frac{k P(k)}{\langle k\rangle} \sigma_{1} I_{k}\right)^{2} d t+\frac{1}{2} \sum_{k=1}^{n} \frac{\Theta^{*}}{\Theta^{2}}\left(\frac{k P(k)}{\langle k\rangle} \sigma_{2} I_{k}\right)^{2} d t \\
= & \mathcal{L} V_{2 k} d t-\left(1-\frac{\Theta^{*}}{\Theta}\right) \sum_{k=1}^{n} \frac{k P(k)}{\langle k\rangle}\left(\sigma_{1} I_{k} d B_{1}-\sigma_{2} I_{k} d B_{2}\right) .
\end{aligned}
$$

Thus

$$
\begin{aligned}
\mathcal{L} & V_{2 k}=\left(1-\frac{\Theta^{*}}{\Theta}\right) \sum_{k=1}^{n} \frac{k P(k)}{\langle k\rangle}\left[\beta k S_{k} \frac{\Theta}{1+b \Theta^{2}}-(v+\gamma+\mu+\lambda) I_{k}\right] \\
& +\frac{1}{2} \sum_{k=1}^{n} \frac{\Theta^{*}}{\Theta^{2}}\left(\frac{k P(k)}{\langle k\rangle} \sigma_{1} I_{k}\right)^{2}+\frac{1}{2} \sum_{k=1}^{n} \frac{\Theta^{*}}{\Theta^{2}}\left(\frac{k P(k)}{\langle k\rangle} \sigma_{2} I_{k}\right)^{2} \\
= & \left(\Theta-\Theta^{*}\right) \sum_{k=1}^{n} \frac{k P(k)}{\langle k\rangle}\left(\beta k \frac{S_{k}}{1+b \Theta^{2}}-\beta k \frac{S_{k}^{*}}{1+b\left(\Theta^{*}\right)^{2}}\right)+\frac{1}{2} \sum_{k=1}^{n} \frac{\Theta^{*}}{\Theta^{2}}\left(\frac{k P(k)}{\langle k\rangle} \sigma_{1} I_{k}\right)^{2} \\
& +\frac{1}{2} \sum_{k=1}^{n} \frac{\Theta^{*}}{\Theta^{2}}\left(\frac{k P(k)}{\langle k\rangle} \sigma_{2} I_{k}\right)^{2} \\
= & \sum_{k=1}^{n} \frac{k P(k)}{\langle k\rangle}\left(\Theta-\Theta^{*}\right)\left[-\left(\beta k \frac{S_{k}^{*}}{1+b\left(\Theta^{*}\right)^{2}}-\beta k \frac{S_{k}}{1+b\left(\Theta^{*}\right)^{2}}+\beta k \frac{S_{k}}{1+b\left(\Theta^{*}\right)^{2}}-\beta k \frac{S_{k}}{1+b \Theta^{2}}\right)\right] \\
& +\frac{1}{2} \sum_{k=1}^{n} \frac{\Theta^{*}}{\Theta^{2}}\left(\frac{k P(k)}{\langle k\rangle} \sigma_{1} I_{k}\right)^{2}+\frac{1}{2} \sum_{k=1}^{n} \frac{\Theta^{*}}{\Theta^{2}}\left(\frac{k P(k)}{\langle k\rangle} \sigma_{2} I_{k}\right)^{2}
\end{aligned}
$$




$$
\begin{aligned}
& =\sum_{k=1}^{n} \frac{k P(k)}{\langle k\rangle} \frac{\beta k}{1+b\left(\Theta^{*}\right)^{2}}\left(S_{k}-S_{k}^{*}\right)\left(\Theta-\Theta^{*}\right) \\
& -\sum_{k=1}^{n} \frac{k P(k)}{\langle k\rangle} \beta k S_{k}\left(\frac{1}{1+b\left(\Theta^{*}\right)^{2}}-\frac{1}{1+b \Theta^{2}}\right)\left(\Theta-\Theta^{*}\right) \\
& +\frac{1}{2} \sum_{k=1}^{n} \frac{\Theta^{*}}{\Theta^{2}}\left(\frac{k P(k)}{\langle k\rangle} \sigma_{1} I_{k}\right)^{2}+\frac{1}{2} \sum_{k=1}^{n} \frac{\Theta^{*}}{\Theta^{2}}\left(\frac{k P(k)}{\langle k\rangle} \sigma_{2} I_{k}\right)^{2} \\
& \leq \sum_{k=1}^{n} \frac{\left\langle k^{2}\right\rangle}{\langle k\rangle} \frac{\beta}{1+b\left(\Theta^{*}\right)^{2}}\left(S_{k}-S_{k}^{*}\right)\left(\Theta-\Theta^{*}\right)+\frac{1}{2} \sum_{k=1}^{n} \frac{\Theta^{*}}{\Theta^{2}}\left(\frac{k P(k)}{\langle k\rangle} \sigma_{1} I_{k}\right)^{2} \\
& +\frac{1}{2} \sum_{k=1}^{n} \frac{\Theta^{*}}{\Theta^{2}}\left(\frac{k P(k)}{\langle k\rangle} \sigma_{2} I_{k}\right)^{2} \\
& =\sum_{k=1}^{n} \frac{k P(k)}{\langle k\rangle}\left(\Theta-\Theta^{*}\right)\left[-\left(\beta k \frac{S_{k}^{*}}{1+b\left(\Theta^{*}\right)^{2}}-\beta k \frac{S_{k}}{1+b\left(\Theta^{*}\right)^{2}}+\beta k \frac{S_{k}}{1+b\left(\Theta^{*}\right)^{2}}-\beta k \frac{S_{k}}{1+b \Theta^{2}}\right)\right] \\
& +\frac{1}{2} \sum_{k=1}^{n} \frac{\Theta^{*}}{\Theta^{2}}\left(\frac{k P(k)}{\langle k\rangle} \sigma_{1} I_{k}\right)^{2}+\frac{1}{2} \sum_{k=1}^{n} \frac{\Theta^{*}}{\Theta^{2}}\left(\frac{k P(k)}{\langle k\rangle} \sigma_{2} I_{k}\right)^{2} \\
& =\sum_{k=1}^{n} \frac{k P(k)}{\langle k\rangle} \frac{\beta k}{1+b\left(\Theta^{*}\right)^{2}}\left(S_{k}-S_{k}^{*}\right)\left(\Theta-\Theta^{*}\right) \\
& -\sum_{k=1}^{n} \frac{k P(k)}{\langle k\rangle} \beta k S_{k}\left(\frac{1}{1+b\left(\Theta^{*}\right)^{2}}-\frac{1}{1+b \Theta^{2}}\right)\left(\Theta-\Theta^{*}\right) \\
& +\frac{1}{2} \sum_{k=1}^{n} \frac{\Theta^{*}}{\Theta^{2}}\left(\frac{k P(k)}{\langle k\rangle} \sigma_{1} I_{k}\right)^{2}+\frac{1}{2} \sum_{k=1}^{n} \frac{\Theta^{*}}{\Theta^{2}}\left(\frac{k P(k)}{\langle k\rangle} \sigma_{2} I_{k}\right)^{2} \\
& \leq \sum_{k=1}^{n} \frac{\left\langle k^{2}\right\rangle}{\langle k\rangle} \frac{\beta}{1+b\left(\Theta^{*}\right)^{2}}\left(S_{k}-S_{k}^{*}\right)\left(\Theta-\Theta^{*}\right) \\
& +\frac{1}{2} \sum_{k=1}^{n} \frac{\Theta^{*}}{\Theta^{2}}\left(\frac{k P(k)}{\langle k\rangle} \sigma_{1} I_{k}\right)^{2}+\frac{1}{2} \sum_{k=1}^{n} \frac{\Theta^{*}}{\Theta^{2}}\left(\frac{k P(k)}{\langle k\rangle} \sigma_{2} I_{k}\right)^{2} .
\end{aligned}
$$

By using the Cauchy-Schwarz Inequality: $\left(\sum_{k=1}^{n} a_{k} b_{k}\right)^{2} \leq\left(\sum_{k=1}^{n} a_{k}^{2}\right)\left(\sum_{k=1}^{n} b_{k}^{2}\right)$, we have

$$
\begin{aligned}
\Theta^{2} & =\left(\sum_{k=1}^{n} \frac{k P(k) I_{k}}{\langle k\rangle}\right)^{2} \\
& =\left(\sum_{k=1}^{n} \frac{k P(k) \sigma_{i} I_{k}}{\langle k\rangle} \frac{1}{\sigma_{i}}\right)^{2} \\
& \leq \sum_{k=1}^{n}\left(\frac{k P(k) \sigma_{i} I_{k}}{\langle k\rangle}\right)^{2}\left(\frac{1}{\sigma_{i}}\right)^{2} \cdot(i=1,2)
\end{aligned}
$$

Thus, we obtain 


$$
\mathcal{L} V_{2 k} \leq \sum_{k=1}^{n} \frac{\left\langle k^{2}\right\rangle}{\langle k\rangle} \frac{\beta}{1+b\left(\Theta^{*}\right)^{2}}\left(S_{k}-S_{k}^{*}\right)\left(\Theta-\Theta^{*}\right)+\frac{1}{2} \sum_{k=1}^{n} \Theta^{*}\left(\frac{1}{\sigma_{1}^{2}}\right)^{-1}+\frac{1}{2} \sum_{k=1}^{n} \Theta^{*}\left(\frac{1}{\sigma_{2}^{2}}\right)^{-1}
$$

Third, we have

$$
\begin{aligned}
d V_{3 k}= & \sum_{k=1}^{n} \xi(k)\left(Q_{k}-Q_{k}^{*}\right)\left[\left(\nu I_{k}-\varepsilon Q_{k}-\mu Q_{k}-\lambda Q_{k}\right) d t-\sigma_{1} Q_{k} d B_{1}(t)-\sigma_{2} Q_{k} d B_{2}(t)\right] \\
& +\frac{1}{2} \sum_{k=1}^{n} \xi(k) \sigma_{1}^{2} Q_{k}^{2}+\frac{1}{2} \sum_{k=1}^{n} \xi(k) \sigma_{2}^{2} Q_{k}^{2} \\
= & \mathcal{L} V_{3 k} d t-\sum_{k=1}^{n} \xi(k)\left(Q_{k}-Q_{k}^{*}\right)\left(\sigma_{1} Q_{k} d B_{1}(t)+\sigma_{2} Q_{k} d B_{2}(t)\right) .
\end{aligned}
$$

Thus

$$
\begin{aligned}
\mathcal{L} V_{3 k}= & \sum_{k=1}^{n} \xi(k)\left(Q_{k}-Q_{k}^{*}\right)\left(\nu I_{k}-\varepsilon Q_{k}-\mu Q_{k}-\lambda Q_{k}\right) \\
& +\frac{1}{2} \sum_{k=1}^{n} \xi(k) \sigma_{1}^{2} Q_{k}^{2}+\frac{1}{2} \sum_{k=1}^{n} \xi(k) \sigma_{2}^{2} Q_{k}^{2} \\
\leq & \sum_{k=1}^{n} \xi(k)\left[\frac{v}{2}\left(I_{k}-I_{k}^{*}\right)^{2}-\left(\varepsilon+\mu+\lambda-\frac{v}{2}-\sigma_{1}^{2}-\sigma_{2}^{2}\right)\left(Q_{k}-Q_{k}^{*}\right)^{2}\right] \\
& +\sum_{k=1}^{n} \xi(k) \sigma_{1}^{2}\left(Q_{k}^{*}\right)^{2}+\sum_{k=1}^{n} \xi(k) \sigma_{2}^{2}\left(Q_{k}^{*}\right)^{2} .
\end{aligned}
$$

Finally, we govern

$$
\begin{aligned}
d V_{4 k}= & \sum_{k=1}^{n} \xi(k)\left(R_{k}-R_{k}^{*}\right)\left[\left(\alpha S_{k}+\gamma I_{k}+\varepsilon Q_{k}-\mu R_{k}\right) d t-\sigma_{1} R_{k} d B_{1}(t)\right] \\
& +\frac{1}{2} \sum_{k=1}^{n} \xi(k) \sigma_{1}^{2} R_{k}^{2} \\
= & \mathcal{L} V_{4 k} d t-\sum_{k=1}^{n} \xi(k)\left(R_{k}-R_{k}^{*}\right) \sigma_{1} R_{k} d B_{1}(t)
\end{aligned}
$$

Thus

$$
\mathcal{L} V_{4 k}=\sum_{k=1}^{n} \xi(k)\left(R_{k}-R_{k}^{*}\right)\left(\alpha S_{k}+\gamma I_{k}+\varepsilon Q_{k}-\mu R_{k}\right)+\frac{1}{2} \sum_{k=1}^{n} \xi(k) \sigma_{1}^{2} R_{k}^{2}
$$




$$
\begin{aligned}
& \leq \sum_{k=1}^{n} \xi(k)\left[\frac{\alpha}{2}\left(S_{k}-S_{k}^{*}\right)^{2}+\frac{\gamma}{2}\left(I_{k}-I_{k}^{*}\right)^{2}+\frac{\varepsilon}{2}\left(Q_{k}-Q_{k}^{*}\right)^{2}\right. \\
& \left.-\left(\mu-\frac{\alpha}{2}-\frac{\gamma}{2}-\frac{\varepsilon}{2}-\sigma_{1}^{2}\right)\left(R_{k}-R_{k}^{*}\right)^{2}\right] \\
& +\sum_{k=1}^{n} \xi(k) \sigma_{1}^{2}\left(R_{k}^{*}\right)^{2} .
\end{aligned}
$$

Accordingly, we have

$$
\begin{aligned}
& \mathcal{L} V\left(S_{1}, I_{1}, Q_{1}, R_{1}, \ldots, S_{n}, I_{n}, Q_{n}, R_{n}\right) \\
& =\mathcal{L} V_{1 k}+\eta^{*} \mathcal{L} V_{2 k}+\mathcal{L} V_{3 k}+\mathcal{L} V_{4 k} \\
& \leq-\left(\alpha+\mu-\sigma_{1}^{2}\right) \sum_{k=1}^{n} \xi(k)\left(S_{k}-S_{k}^{*}\right)^{2}-\left(v+\gamma+\mu+\lambda-\sigma_{1}^{2}-\sigma_{2}^{2}\right) \sum_{k=1}^{n} \xi(k)\left(I_{k}-I_{k}^{*}\right)^{2}-(\alpha+2 \mu+v \\
& +\gamma+\lambda)\left(S_{k}-S_{k}^{*}\right)\left(\Theta-\Theta^{*}\right)+\sum_{k=1}^{n} \xi(k)\left[\sigma_{1}^{2}\left(S_{k}^{*}\right)^{2}+\sigma_{1}^{2}\left(I_{k}^{*}\right)^{2}+\sigma_{2}^{2}\left(I_{k}^{*}\right)^{2}\right] \\
& +\frac{(\alpha+2 \mu+v+\gamma+\lambda)\left(1+b\left(\Theta^{*}\right)^{2}\right)\langle k\rangle}{\beta\left\langle k^{2}\right\rangle}\left[\sum_{k=1}^{n} \frac{\left\langle k^{2}\right\rangle}{\langle k\rangle} \frac{\beta}{1+b\left(\Theta^{*}\right)^{2}}\left(S_{k}-S_{k}^{*}\right)\left(\Theta-\Theta^{*}\right)+\frac{1}{2} \sum_{k=1}^{n} \Theta^{*}\left(\frac{1}{\sigma_{1}^{2}}\right)^{-1}\right. \\
& \left.+\frac{1}{2} \sum_{k=1}^{n} \Theta^{*}\left(\frac{1}{\sigma_{2}^{2}}\right)^{-1}\right]+\sum_{k=1}^{n} \xi(k)\left[\frac{v}{2}\left(I_{k}-I_{k}^{*}\right)^{2}-\left(\varepsilon+\mu+\lambda-\frac{v}{2}-\sigma_{1}^{2}-\sigma_{2}^{2}\right)\left(Q_{k}-Q_{k}^{*}\right)^{2}\right]+\sum_{k=1}^{n} \xi(k) \sigma_{1}^{2} \\
& \left(Q_{k}^{*}\right)^{2}+\sum_{k=1}^{n} \xi(k) \sigma_{2}^{2}\left(Q_{k}^{*}\right)^{2}+\sum_{k=1}^{n} \xi(k)\left[\frac{\alpha}{2}\left(S_{k}-S_{k}^{*}\right)^{2}+\frac{\gamma}{2}\left(I_{k}-I_{k}^{*}\right)^{2}+\frac{\varepsilon}{2}\left(Q_{k}-Q_{k}^{*}\right)^{2}-\left(\mu-\frac{\alpha}{2}-\frac{\gamma}{2}-\frac{\varepsilon}{2}-\sigma_{1}^{2}\right)\right. \\
& \left.\left(R_{k}-R_{k}^{*}\right)^{2}\right]+\sum_{k=1}^{n} \xi(k) \sigma_{1}^{2}\left(R_{k}^{*}\right)^{2} \\
& =-\sum_{k=1}^{n} \xi(k)\left[\left(\alpha+\mu-\frac{\alpha}{2}-\sigma_{1}^{2}\right)\left(S_{k}-S_{k}^{*}\right)^{2}+\left(\frac{\nu}{2}+\frac{\gamma}{2}+\mu+\lambda-\sigma_{1}^{2}-\sigma_{2}^{2}\right)\left(I_{k}-I_{k}^{*}\right)^{2}+\left(\frac{\varepsilon}{2}+\mu+\lambda-\frac{v}{2}\right.\right. \\
& \left.\left.-\sigma_{1}^{2}-\sigma_{2}^{2}\right)\left(Q_{k}-Q_{k}^{*}\right)^{2}+\left(\frac{v}{2}+\frac{\gamma}{2}+\mu+\lambda-\sigma_{1}^{2}-\sigma_{2}^{2}\right)\left(R_{k}-R_{k}^{*}\right)^{2}\right]+\sum_{k=1}^{n} \xi(k)\left[\sigma_{1}^{2}\left(S_{k}^{*}\right)^{2}+\left(\sigma_{1}^{2}+\sigma_{2}^{2}\right)\left(I_{k}^{*}\right)^{2}\right. \\
& \left.+\left(\sigma_{1}^{2}+\sigma_{2}^{2}\right)\left(Q_{k}^{*}\right)^{2}+\sigma_{1}^{2}\left(R_{k}^{*}\right)^{2}+\frac{1}{2} \frac{(\alpha+2 \mu+v+\gamma+\lambda)\left(1+b\left(\Theta^{*}\right)^{2}\right)\langle k\rangle}{\beta\left\langle k^{2}\right\rangle}\left(\sigma_{1}^{2}+\sigma_{2}^{2}\right) I_{k}^{*}\right] \\
& =-\sum_{k=1}^{n} \xi(k)\left[\left(\alpha+\mu-\frac{\alpha}{2}-\sigma_{1}^{2}\right)\left(S_{k}-S_{k}^{*}\right)^{2}+\left(\frac{\nu}{2}+\frac{\gamma}{2}+\mu+\lambda-\sigma_{1}^{2}-\sigma_{2}^{2}\right)\left(I_{k}-I_{k}^{*}\right)^{2}+\left(\frac{\varepsilon}{2}+\mu+\lambda-\frac{v}{2}\right.\right. \\
& \left.\left.-\sigma_{1}^{2}-\sigma_{2}^{2}\right)\left(Q_{k}-Q_{k}^{*}\right)^{2}+\left(\mu-\frac{\alpha}{2}-\frac{\gamma}{2}-\frac{\varepsilon}{2}-\sigma_{1}^{2}\right)\left(R_{k}-R_{k}^{*}\right)^{2}\right]+F .
\end{aligned}
$$

one can easily find that the ellipsoid

$$
\begin{aligned}
& \sum_{k=1}^{n} \xi(k)\left[\left(\alpha+\mu-\frac{\alpha}{2}-\sigma_{1}^{2}\right)\left(S_{k}-S_{k}^{*}\right)^{2}+\left(\frac{v}{2}+\frac{\gamma}{2}+\mu+\lambda-\sigma_{1}^{2}-\sigma_{2}^{2}\right)\left(I_{k}-I_{k}^{*}\right)^{2}\right. \\
& \left.+\left(\frac{\varepsilon}{2}+\mu+\lambda-\frac{v}{2}-\sigma_{1}^{2}-\sigma_{2}^{2}\right)\left(Q_{k}-Q_{k}^{*}\right)^{2}+\left(\mu-\frac{\alpha}{2}-\frac{\gamma}{2}-\frac{\varepsilon}{2}-\sigma_{1}^{2}\right)\left(R_{k}-R_{k}^{*}\right)^{2}\right]=F .
\end{aligned}
$$


lie in the positive zone of $\mathbb{R}_{+}^{4 n}$. Consequently, there exists a constant $C>0$ and a compact set $U \subset \mathbb{R}_{+}^{4 n}$ such that for any $\left(S_{1}, I_{1}, Q_{1}, R_{1}, \cdots, S_{n}, I_{n}, Q_{n}, R_{n}\right) \in$ $\mathbb{R}_{+}^{4 n} \backslash U$, we have

$$
\begin{aligned}
& \sum_{k=1}^{n} \xi(k)\left[\left(\alpha+\mu-\frac{\alpha}{2}-\sigma_{1}^{2}\right)\left(S_{k}-S_{k}^{*}\right)^{2}+\left(\frac{\nu}{2}+\frac{\gamma}{2}+\mu+\lambda-\sigma_{1}^{2}-\sigma_{2}^{2}\right)\left(I_{k}-I_{k}^{*}\right)^{2}\right. \\
& \left.+\left(\frac{\varepsilon}{2}+\mu+\lambda-\frac{\nu}{2}-\sigma_{1}^{2}-\sigma_{2}^{2}\right)\left(Q_{k}-Q_{k}^{*}\right)^{2}+\left(\mu-\frac{\alpha}{2}-\frac{\gamma}{2}-\frac{\varepsilon}{2}-\sigma_{1}^{2}\right)\left(R_{k}-R_{k}^{*}\right)^{2}\right] \geq F+C .
\end{aligned}
$$

Accordingly, we derive $\mathcal{L} V \leq-C, \forall\left(S_{1}, I_{1}, Q_{1}, R_{1}, \cdots, S_{n}, I_{n}, Q_{n}, R_{n}\right) \in$ $\mathbb{R}_{+}^{4 n} \backslash U$, this manifests that condition $A_{2}$ of Lemma 4.3 is satisfied. To sum up, stochastic model (2) has a unique stationary distribution and the ergodic property. This completes the proof.

As for the problem that there is no endemic equilibrium in the stochastic model (2), we will explain it further below.

Theorem 7.2 When $R_{0}>1$, if the conditions $\omega_{i}>0(i=1,2,3,4), F<$ $\min \left\{\omega_{1}\left(S_{k}^{*}\right)^{2}, \omega_{2}\left(I_{k}^{*}\right)^{2}, \omega_{3}\left(Q_{k}^{*}\right)^{2}, \omega_{4}\left(R_{k}^{*}\right)^{2}\right\}, M=\min \left\{\omega_{1}, \omega_{2}, \omega_{3}, \omega_{4}\right\}$ are satisfied, then the solution $\left(S_{1}(t), I_{1}(t), Q_{1}(t), R_{1}(t), \cdots, S_{n}(t), I_{n}(t), Q_{n}(t), R_{n}(t)\right)$ of stochastic model (2)with initial value $\left(S_{1}(0), I_{1}(0), Q_{1}(0), R_{1}(0), \cdots, S_{n}(0)\right.$, $\left.I_{n}(0), Q_{n}(0), R_{n}(0)\right)$ has the following property:

$$
\lim _{t \rightarrow \infty} \sup \frac{1}{t} E \int_{0}^{t} \sum_{k=1}^{n} \xi(k)\left[\left(S_{k}(r)-S_{k}^{*}\right)^{2}+\left(I_{k}(r)-I_{k}^{*}\right)^{2}+\left(Q_{k}(r)-Q_{k}^{*}\right)^{2}+\left(R_{k}(r)-R_{k}^{*}\right)^{2}\right] d r<\frac{F}{M} .
$$

where $\left(S_{1}^{*}, I_{1}^{*}, Q_{1}^{*}, R_{1}^{*}, \cdots, S_{n}^{*}, I_{n}^{*}, Q_{n}^{*}, R_{n}^{*}\right)$ is the endemic equilibrium of deterministic model (1),

$$
\begin{aligned}
\omega_{1}= & \sum_{k=1}^{n} \xi(k)\left(\alpha+\mu-\frac{\alpha}{2}-\sigma_{1}^{2}\right), \\
\omega_{2}= & \sum_{k=1}^{n} \xi(k)\left(\frac{v}{2}+\frac{\gamma}{2}+\mu+\lambda-\sigma_{1}^{2}-\sigma_{2}^{2}\right), \\
\omega_{3}= & \sum_{k=1}^{n} \xi(k)\left(\frac{\varepsilon}{2}+\mu+\lambda-\frac{v}{2}-\sigma_{1}^{2}-\sigma_{2}^{2}\right), \\
\omega_{4}= & \sum_{k=1}^{n} \xi(k)\left(\mu-\frac{\alpha}{2}-\frac{\gamma}{2}-\frac{\varepsilon}{2}-\sigma_{1}^{2}\right), \\
F= & \sum_{k=1}^{n} \xi(k)\left[l_{1}\left(S_{k}^{*}\right)^{2}+l_{2}\left(I_{k}^{*}\right)^{2}+l_{2}\left(Q_{k}^{*}\right)^{2}+l_{1}\left(R_{k}^{*}\right)^{2}\right. \\
& \left.+\frac{1}{2} \frac{(\alpha+2 \mu+v+\gamma+\lambda)\left(1+b\left(\Theta^{*}\right)^{2}\right)\langle k\rangle}{\beta\left\langle k^{2}\right\rangle}\left(\sigma_{1}^{2}+\sigma_{2}^{2}\right) I_{k}^{*}\right],
\end{aligned}
$$




$$
l_{1}=\sigma_{1}^{2}, \quad l_{2}=\sigma_{1}^{2}+\sigma_{2}^{2}, \quad \xi(k)=\frac{1}{\langle k\rangle} \sum_{k=1}^{n} k P(k) .
$$

Proof

$$
\begin{aligned}
\omega_{1}= & \sum_{k=1}^{n} \xi(k)\left(\alpha+\mu-\frac{\alpha}{2}-\sigma_{1}^{2}\right), \\
\omega_{2}= & \sum_{k=1}^{n} \xi(k)\left(\frac{v}{2}+\frac{\gamma}{2}+\mu+\lambda-\sigma_{1}^{2}-\sigma_{2}^{2}\right), \\
\omega_{3}= & \sum_{k=1}^{n} \xi(k)\left(\frac{\varepsilon}{2}+\mu+\lambda-\frac{v}{2}-\sigma_{1}^{2}-\sigma_{2}^{2}\right), \\
\omega_{4}= & \sum_{k=1}^{n} \xi(k)\left(\mu-\frac{\alpha}{2}-\frac{\gamma}{2}-\frac{\varepsilon}{2}-\sigma_{1}^{2}\right), \\
F= & \sum_{k=1}^{n} \xi(k)\left[l_{1}\left(S_{k}^{*}\right)^{2}+l_{2}\left(I_{k}^{*}\right)^{2}+l_{2}\left(Q_{k}^{*}\right)^{2}+l_{1}\left(R_{k}^{*}\right)^{2}\right. \\
& \left.+\frac{1}{2} \frac{(\alpha+2 \mu+v+\gamma+\lambda)\left(1+b\left(\Theta^{*}\right)^{2}\right)\langle k\rangle}{\beta\left\langle k^{2}\right\rangle}\left(\sigma_{1}^{2}+\sigma_{2}^{2}\right) I_{k}^{*}\right], \\
l_{1}= & \sigma_{1}^{2}, \quad l_{2}=\sigma_{1}^{2}+\sigma_{2}^{2}, \quad \xi(k)=\frac{1}{\langle k\rangle} \sum_{k=1}^{n} k P(k) .
\end{aligned}
$$

Define $\mathrm{C}^{2}$-function $V: \Gamma \rightarrow \mathbb{R}_{+}$by

$$
\begin{aligned}
V( & \left.S_{1}, I_{1}, Q_{1}, R_{1}, \cdots S_{n}, I_{n}, Q_{n}, R_{n}\right) \\
= & \frac{1}{2} \sum_{k=1}^{n} \xi(k)\left(S_{k}-S_{k}^{*}+I_{k}-I_{k}^{*}\right)^{2}+\eta^{*}\left(\Theta-\Theta^{*}-\Theta^{*} \ln \frac{\Theta}{\Theta^{*}}\right) \\
& +\frac{1}{2} \sum_{k=1}^{n} \xi(k)\left(Q_{k}-Q_{k}^{*}\right)^{2}+\frac{1}{2} \sum_{k=1}^{n} \xi(k)\left(R_{k}-R_{k}^{*}\right)^{2} \\
= & : V_{1 k}+\eta^{*} V_{2 k}+V_{3 k}+V_{4 k} .
\end{aligned}
$$

where $\eta^{*}=\frac{(\alpha+2 \mu+\nu+\gamma+\lambda)\left(1+b\left(\Theta^{*}\right)^{2}\right)\langle k\rangle}{\beta\left\langle k^{2}\right\rangle}$.

From Theorem 7.1, we can obtain

$$
\begin{aligned}
& \mathcal{L} V\left(S_{1}, I_{1}, Q_{1}, R_{1}, \ldots, S_{n}, I_{n}, Q_{n}, R_{n}\right) \\
& \quad \leq-\sum_{k=1}^{n} \xi(k)\left[\left(\alpha+\mu-\frac{\alpha}{2}-\sigma_{1}^{2}\right)\left(S_{k}-S_{k}^{*}\right)^{2}+\left(\frac{\nu}{2}+\frac{\gamma}{2}+\mu+\lambda-\sigma_{1}^{2}-\sigma_{2}^{2}\right)\left(I_{k}-I_{k}^{*}\right)^{2}\right.
\end{aligned}
$$




$$
\left.+\left(\frac{\varepsilon}{2}+\mu+\lambda-\frac{\nu}{2}-\sigma_{1}^{2}-\sigma_{2}^{2}\right)\left(Q_{k}-Q_{k}^{*}\right)^{2}+\left(\mu-\frac{\alpha}{2}-\frac{\gamma}{2}-\frac{\varepsilon}{2}-\sigma_{1}^{2}\right)\left(R_{k}-R_{k}^{*}\right)^{2}\right]+F .
$$

By integrating both sides of Eq. (12) and taking the expected value as follows:

$0 \leq E\left[V\left(S_{1}, I_{1}, Q_{1}, R_{1}, \ldots, S_{n}, I_{n}, Q_{n}, R_{n}\right)\right]$

$<V\left(S_{1}(0), I_{1}(0), Q_{1}(0), R_{1}(0), \ldots, S_{n}(0), I_{n}(0), Q_{n}(0), R_{n}(0)\right)$

$$
\begin{aligned}
& -E \int_{0}^{t} \sum_{k=1}^{n} \xi(k)\left[\left(\alpha+\mu-\frac{\alpha}{2}-\sigma_{1}^{2}\right)\left(S_{k}(r)-S_{k}^{*}\right)^{2}+\left(\frac{v}{2}+\frac{\gamma}{2}+\mu+\lambda-\sigma_{1}^{2}-\sigma_{2}^{2}\right)\left(I_{k}(r)-I_{k}^{*}\right)^{2}\right. \\
& \left.\left(\frac{\varepsilon}{2}+\mu+\lambda-\frac{v}{2}-\sigma_{1}^{2}-\sigma_{2}^{2}\right)\left(Q_{k}(r)-Q_{k}^{*}\right)^{2}+\left(\mu-\frac{\alpha}{2}-\frac{\gamma}{2}-\frac{\varepsilon}{2}-\sigma_{1}^{2}\right)\left(R_{k}(r)-R_{k}^{*}\right)^{2}+F\right] d r .
\end{aligned}
$$

that is

$$
\begin{aligned}
& E \int_{0}^{t} \sum_{k=1}^{n} \xi(k)\left[\left(\alpha+\mu-\frac{\alpha}{2}-\sigma_{1}^{2}\right)\left(S_{k}(r)-S_{k}^{*}\right)^{2}+\left(\frac{v}{2}+\frac{\gamma}{2}+\mu+\lambda-\sigma_{1}^{2}-\sigma_{2}^{2}\right)\left(I_{k}(r)-I_{k}^{*}\right)^{2}\right. \\
& \left.\left(\frac{\varepsilon}{2}+\mu+\lambda-\frac{v}{2}-\sigma_{1}^{2}-\sigma_{2}^{2}\right)\left(Q_{k}(r)-Q_{k}^{*}\right)^{2}+\left(\mu-\frac{\alpha}{2}-\frac{\gamma}{2}-\frac{\varepsilon}{2}-\sigma_{1}^{2}\right)\left(R_{k}(r)-R_{k}^{*}\right)^{2}\right] d r \\
& <V\left(S_{1}(0), I_{1}(0), Q_{1}(0), R_{1}(0), \cdots, S_{n}(0), I_{n}(0), Q_{n}(0), R_{n}(0)\right)+F t .
\end{aligned}
$$

Accordingly,

$$
\begin{aligned}
& \lim _{t \rightarrow \infty} \sup \frac{1}{t} E \int_{0}^{t} \sum_{k=1}^{n} \xi(k)\left[\left(\alpha+\mu-\frac{\alpha}{2}-\sigma_{1}^{2}\right)\left(S_{k}(r)-S_{k}^{*}\right)^{2}+\left(\frac{\nu}{2}+\frac{\gamma}{2}+\mu+\lambda-\sigma_{1}^{2}-\sigma_{2}^{2}\right)\left(I_{k}(r)-I_{k}^{*}\right)^{2}\right. \\
& \left.\left(\frac{\varepsilon}{2}+\mu+\lambda-\frac{\nu}{2}-\sigma_{1}^{2}-\sigma_{2}^{2}\right)\left(Q_{k}(r)-Q_{k}^{*}\right)^{2}+\left(\mu-\frac{\alpha}{2}-\frac{\gamma}{2}-\frac{\varepsilon}{2}-\sigma_{1}^{2}\right)\left(R_{k}(r)-R_{k}^{*}\right)^{2}\right] d r<F .
\end{aligned}
$$

It is easy to obtain that $M>0$ from the known conditions.

Therefore

$$
\lim _{t \rightarrow \infty} \sup \frac{1}{t} E \int_{0}^{t} \sum_{k=1}^{n} \xi(k)\left[\left(S_{k}(r)-S_{k}^{*}\right)^{2}+\left(I_{k}(r)-I_{k}^{*}\right)^{2}+\left(Q_{k}(r)-Q_{k}^{*}\right)^{2}+\left(R_{k}(r)-R_{k}^{*}\right)^{2}\right] d r<\frac{F}{M}
$$

Remark 7.1 According to Theorem 7.2, we acquire that the solution of stochastic model (2) fluctuates around the endemic equilibrium $E^{*}$ of deterministic model (1), with the values of $\sigma_{i}^{2}(i=1,2)$ decreasing, the solution of stochastic model (2) will be close to $E^{*}$, the disease will persist.

Remark 7.2 From Theorem 7.2, we can see that the solution of system (2) will oscillate around the endemic equilibrium $E^{*}$ under $R_{0}>1$. From $I_{k}^{*}=$ $\frac{\mu \beta k \Theta^{*}}{(v+\gamma+\mu+\lambda)\left\{(\alpha+\mu)\left[1+b\left(\Theta^{*}\right)^{2}\right]+\beta k \Theta^{*}\right\}}$, it is obtained that $I_{k}^{*}$ decreases with the increase of quarantine rate $v$. Accordingly, quarantine measures reduce the final infection scale. 


\section{Numerical experiments}

This section is carried out some numerical experiments with the aim of illustrating the validity of our obtained theoretical results. By using preferential attachment mechanism in [8], a scale-free network Q with the number of nodes on network is $N=100$ and $P(k)=(r-1) m^{r-1} k^{-r},(m=1, r=3)$, one can easily obtained that $\langle k\rangle=3.27,\left\langle k^{2}\right\rangle=9.04$.

Example 8.1 Based on Q, the basic reproduction number $R_{0}=\frac{\left\langle k^{2}\right\rangle}{\langle k\rangle} \frac{\beta \mu}{(\mu+\alpha)(v+\gamma+\lambda+\mu)} \approx$ 0.95 is given with the parameters $\beta=0.54, \alpha=0.0088, \mu=0.0006, v=0.04$, $\gamma=0.04, \lambda=0.02, \varepsilon=0.001, b=3$. Figure 2 demonstrates that there always exists a disease-free equilibrium in Theorem 3.1. In addition, the intensities of noises $\sigma_{1}=\sigma_{2}=0.02$, Fig. 3 shows when $R_{0}<1$, the disease becomes extinct, which demonstrates the validity of Theorem 6.1 .

Example 8.2 Based on Q, the basic reproduction number $R_{0}=\frac{\left\langle k^{2}\right\rangle}{\langle k\rangle} \frac{\beta \mu}{(\mu+\alpha)(v+\gamma+\lambda+\mu)} \approx$ $2.36>1$ is given with the parameters $\beta=0.55, \alpha=0.023, \mu=0.02, v=0.1$, $\gamma=0.1, \lambda=0.08, \varepsilon=0.001, b=3$. Figure 4 proves that the disease will extinct if $\sigma_{1}=\sigma_{2}=0.3$ enough large, regardless of whether $R_{0}$ is greater than one.

Example 8.3 Based on Q, the basic reproduction number $R_{0}=\frac{\left\langle k^{2}\right\rangle}{\langle k\rangle} \frac{\beta \mu}{(\mu+\alpha)(v+\gamma+\lambda+\mu)} \approx$ $7.15>1$ is given with the parameters $\beta=0.75, \alpha=0.01, \mu=0.0235, v=0.08$, $\gamma=0.05, \lambda=0.05, \varepsilon=0.001, b=3$, which satisfy conditions of Theorem 3.1 and Theorem 7.1. Figure 5 demonstrates the validity of Theorem 3.1

By showing the existence of endemic equilibrium. In addition, the intensities of noises $\sigma_{1}=0.02, \sigma_{2}=0.02$, Fig. 6 manifests that solution of stochastic model

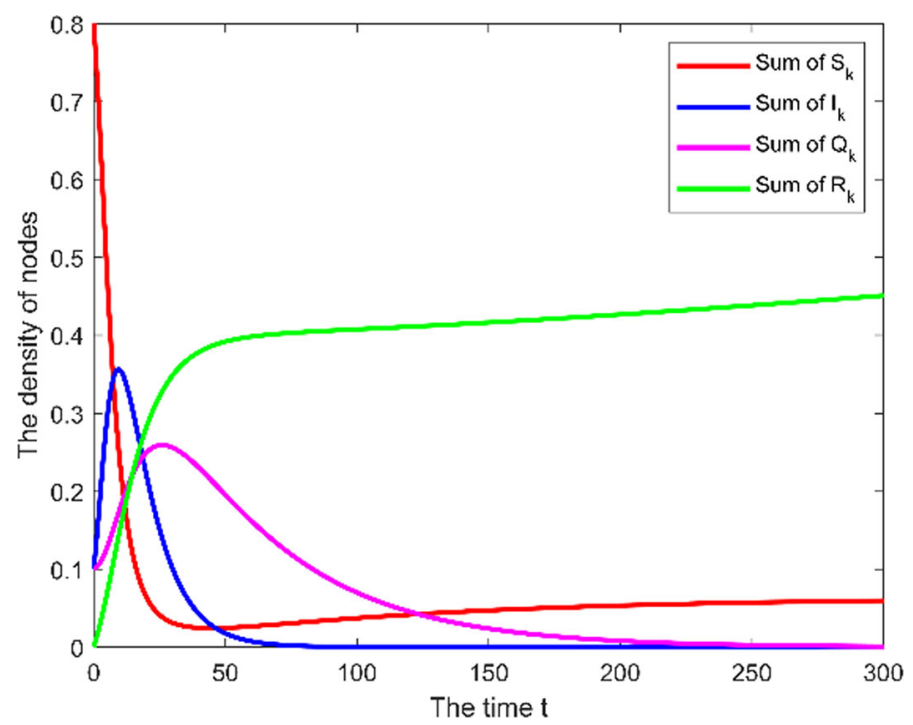

Fig. 2 Trajectories of system (1) with $R_{0}<1$. 


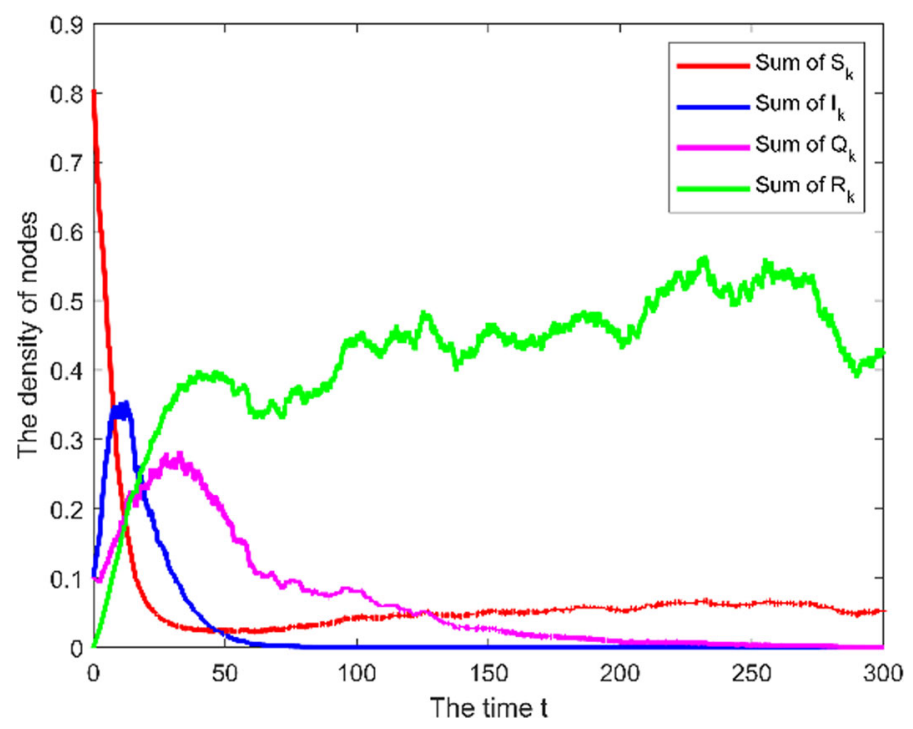

Fig. 3 Trajectories of system (2) with $R_{0}<1$ and $\sigma_{1}=\sigma_{2}=0.02$.

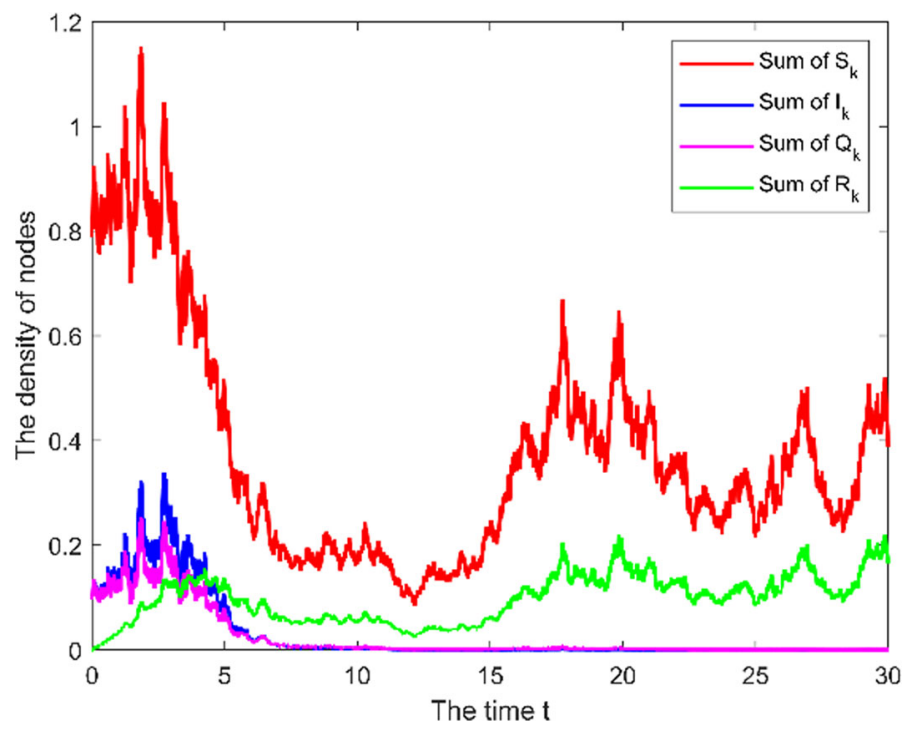

Fig. 4 Trajectories of system (2) with $R_{0}>1$ and $\sigma_{1}=\sigma_{2}=0.3$.

(2) has a unique stationary distribution and ergodic property, which demonstrates the validity of Theorem 7.1 . 


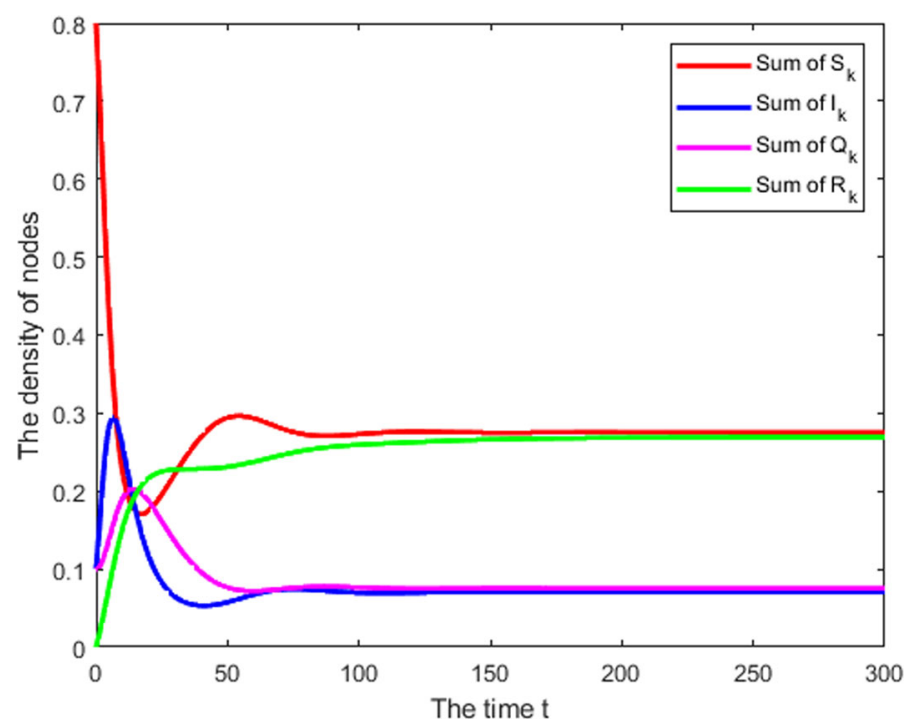

Fig. 5 Trajectories of system (1) with $R_{0}>1$.

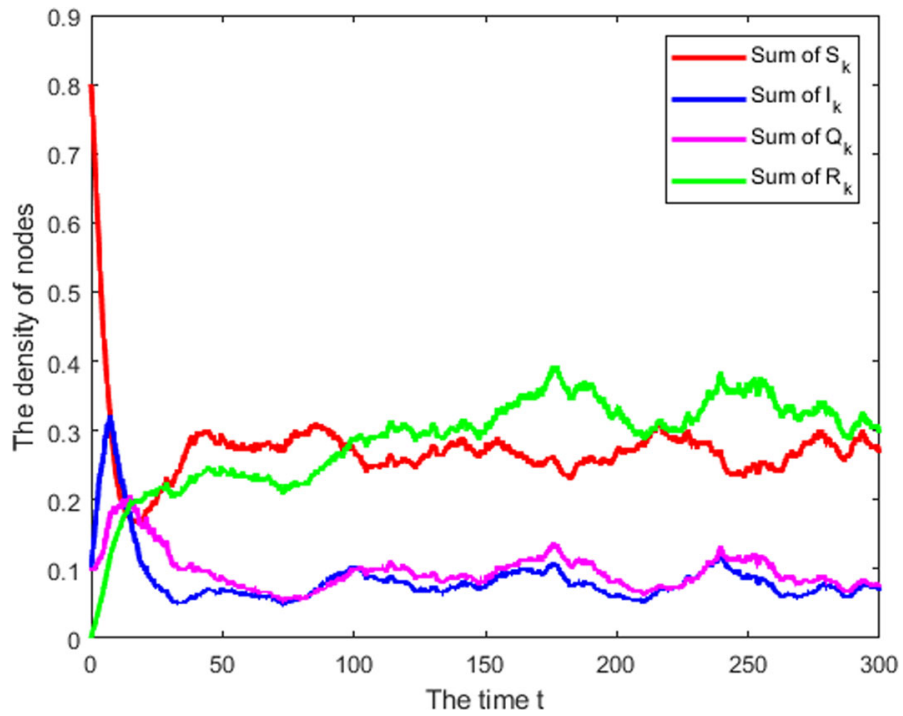

Fig. 6 Trajectories of system (2) with $R_{0}>1$ and $\sigma_{1}=\sigma_{2}=0.02$.

\section{Conclusions}

It is well known that traditional compartment models assume that individuals are uniformly mixed, that is, an individual has equal opportunities to contact with any other 
individual in unit time. However, due to the difference of people's living environment, education level, interests, hobbies and status, their social circle and scope of activities will be significantly different, so the possibility of spreading diseases is also different. With the development of complex networks, some scholars have found that social networks exhibit scale-free characteristics, accordingly, this paper discusses the dynamic behavior of epidemic on scale-free networks, which makes up for the lack of uniform mixing of traditional compartment model. In addition, when the disease begins to spread, news of the disease will spread rapidly, and people who get the news will take preventive measures to reduce the infection rate, obviously, non-monotone incidence rate can reveal this phenomenon more accurately. Similarly, due to resource constraints, population migration, diseases threat and so on, the population size will also change. Furthermore, when an epidemic breaks out, the government usually takes quarantine measures to protect people's lives and property. In fact, the spread of disease is inevitably affected by various random factors, in order to comprehensively explore the influence of the above factors on the dynamic behavior of disease, we presented a stochastic SIQR epidemic model with demographics and non-monotone incidence rate on scale-free networks. Another aim of this paper is to investigate the extinction and stationary distribution of a stochastic SIQR epidemic model with demographics and non-monotone incidence rate on scale-free networks, which introduces stochastic perturbations to natural death and mortality from diseases of the deterministic model (1). At first, the formula of the basic reproduction number for the model (1) is obtained and the existence of equilibrium is demonstrated, the obtained results show that epidemic threshold depends on the level of heterogeneity of the network $\left(\left\langle k^{2}\right\rangle /\langle k\rangle\right)$ and the quarantined rate $(v)$, then if the heterogeneity of network increases, the risk of diseases outbreak rises, if the quarantined rate increases, the corresponding basic reproduction number $R_{0}$ and the final infection size $I_{k}^{*}$ decrease. Next, for the stochastic model (2), existence and uniqueness of global positive solution is proved by constructing appropriate Lyapunov functions. In addition, sufficient conditions for diseases extinction and existence of ergodic stationary distribution is manifested, we found that when $R_{0} \leq 1, I_{k}(t)$ tends to zero almost surely, that is to say, the diseases extinct eventually, what is more, the diseases will die out eventually with probability one if $\sigma_{1}^{2}+\sigma_{2}^{2}$ enough large, regardless of whether $R_{0}$ is greater than one; when $R_{0}>1$, under some certain conditions, stochastic model (2) has a unique stationary distribution and ergodic property, that is, the disease will persist.

The research of this work enriches the theory of propagation dynamics on scalefree networks and provide us valuable guidance to prevent the spread of epidemic. In addition, because there are many similarities on spreading behaviors of computer virus, public opinions and rumors with infectious diseases, some methods used here are also applicable to study some problems in those fields.

There are some meaningful works deserve further investigations. In real life, individuals of different ages have great differences in resistance to epidemics [32, 33], accordingly, the epidemic model considering age structure deserves further discussion. In fact, due to the difference of individual health status and the change of natural environment, the infection period and incubation period of most pathogens will also change accordingly, so it is necessary to introduce distributed delay into the model. Furthermore, for some unexpected phenomenon (earthquake, typhoon, and so on), 
random continuous noise (Brownian motion) cannot describe these phenomena accurately, therefore, it is fantastic to introduce a more general lévy process to describe these abnormal phenomena. In addition, epidemic transmission on the actual contact network and early warning information transmission on the information communication network restricted each other, compared with the single-layer network, the multi-layer network can more accurately simulate the real social system. Accordingly, the research on the propagation dynamics based on the multi-layer network may have more theoretical value and practical significance.

Acknowledgements The authors thank the anonymous reviewers for their insightful comments and suggestions, which is valuable for improving the accuracy of the manuscript. This work is supported by the National Natural Science Foundation of China (grant 11361044), the Natural Science Foundation of Ningxia (Grant 2021AAC03030), the Graduate Innovation Program of Ning Xia University (grant GIP2020060).

\section{References}

1. Hethcote, H.: The mathematics of infectious diseases. SIAM Rev. 42, 599-653 (2000)

2. Zhu, G., Chen, G., Zhang, H., Fu, X.: Propagation dynamics of an epidemic model with infective media connecting two separated networks of populations. Commun. Nonlinear Sci. Numer. Simul. 20, 240-249 (2015)

3. Djilali, S., Ghanbari, B., Bentout, S., Mezouaghi, A.: Turing-Hopf bifurcation in a diffusive musselalgae model with time-fractional-order derivative. Chaos Solitons Fractals 138, 109954 (2020)

4. Pgda, B., Cckc, D., Rmse, F.: Determining the optimal strategy for reopening schools, the impact of test and trace interventions, and the risk of occurrence of a second COVID-19 epidemic wave in the UK: a modelling study-ScienceDirect. Lancet Child Adolescent Health 4, 817-827 (2020)

5. Bentout, S., Chekroun, A., Kuniya, T.: Parameter estimation and prediction for coronavirus disease outbreak 2019 (COVID-19) in Algeria. AIMS Public Health 7, 306-318 (2020)

6. Liljeros, F., Edling, C.R., Amaral, L.A.N.: The web of human sexual contacts. Nature 411, 907-908 (2001)

7. Wang, Z., Andrews, M., Wu, Z., Wang, L., Bauch, C.: Coupled disease-behavior dynamics on complex networks: a review. Phys. Life Rev. 15, 1-29 (2015)

8. Barabási, A.L., Albert, R.: Emergence of scaling in random networks. Science 286, 509-512 (1999)

9. Albert, R., Jeong, H., Barabási, A.L.: Diameter of the world wide web. Nature 401, 130-131 (1999)

10. Faloutsos, M., Faloutsos, P., Faloutsos, C.: On power-law relationships of the internet topology. Comput. Commun. Rev. 29, 251-262 (1999)

11. Geraldine, B., Antoine, M.: Social interactions and the prophylaxis of SI epidemics on networks. J. Math. Econ. 93, 102486 (2021)

12. Romualdo, P.S., Alessandro, V.: Epidemic spreading in scale-free networks. Phys. Rev. Lett. 86, 3200-3203 (2000)

13. Wei, X., Xu, G., Liu, L.: Global stability of endemic equilibrium of an epidemic model with birth and death on complex networks. Physica A 477, 8-84 (2017)

14. Li, T., Wang, Y., Guan, Z.: Spreading dynamics of a SIQRS epidemic model on scale-free networks. Commun. Nonlinear Sci. Numer. Simul. 19, 686-692 (2014)

15. Huang, S., Chen, F., Chen, L.: Global dynamics of a network-based SIQRS epidemic model with demographics and vaccination. Commun. Nonlinear Sci. Numer. Simul. 43, 296-310 (2017)

16. Meng, X., Cai, Z., Si, S.: Analysis of epidemic vaccination strategies on heterogeneous networks: based on SEIRV model and evolutionary game. Appl. Math. Comput. 403, 126 (2021)

17. Kang, H., Fu, X.: Epidemic spreading and global stability of an SIS model with an infective vector on complex networks. Commun. Nonlinear Sci. Numer. Simul. 27, 30-39 (2015)

18. Xiao, D., Ruan, S.: Global analysis of an epidemic model with non-monotone incidence rate. Math. Biosci. 208, 419-429 (2007)

19. Chun-Hsien, L.: Dynamics of a network-based SIS epidemic model with non-monotone incidence rate. Physica A 427, 234-243 (2015) 
20. Zhao, D.: Study on the threshold of a stochastic SIR epidemic model and its extensions. Commun. Nonlinear Sci. Numer. Simul. 38, 172-177 (2016)

21. Liu, Q., Jiang, D., Shi, N.: Stationary distribution and extinction of a stochastic SEIR epidemic model with standard incidence. Physica A 469, 510-517 (2017)

22. Zhang, Y., Li, Y.: Evolutionary dynamics of stochastic SEIR models with migration and human awareness in complex networks. Complexity 2020, 3768083 (2020)

23. Zhao, R., Liu, Q., Sun, M.: Dynamical behavior of a stochastic SIQS epidemic model on scale-free networks. J. Appl. Math. Comput. 9, 1-26 (2021)

24. Chen, Y., Wen, B., Teng, Z.: The global dynamics for a stochastic SIS epidemic model with isolation. Physica A 492, 1604-1624 (2018)

25. Pan, L.J., Cao, J.D., Ahmed, A.: Stability of reaction-diffusion systems with stochastic switching. Nonlinear Anal. Model. Control 24, 315-331 (2019)

26. Fatini, M.E., Pettersson, R., Sekkak, I.: A stochastic analysis for a triple delayed SIQR epidemic model with vaccination and elimination strategies. J. Appl. Math. Comput. 64, 781-805 (2020)

27. Bentout, S., Chen, Y., Djilali, S.: Global dynamics of an SEIR model with two age structures and a nonlinear incidence. Acta Appl. Math. 171, 1-27 (2021)

28. Has'miniskii, R.Z.: Stochastic Stability of Differential Equations. Springer, Berlin (1980)

29. ItÔ, K.: On Stochastic Differential Equations. On Stochastic Differential Equations. American Mathematical Society (1951)

30. Chen, F.: On a nonlinear nonautonomous predator-prey model with diffusion and distributed delay. Appl. Comput. Math. 180, 33-49 (2005)

31. Friedman, A.: Stochastic Differential Equations and Applications. Academic Press (1976)

32. Soufiane, B., Touaoula, T.M.: Global analysis of an infection age model with a class of nonlinear incidence rates. J. Math. Anal. Appl. 434, 1211-1239 (2017)

33. Bentout, S., Tridane, A., Djilali, S., Touaoula, T.M.: Age-structured modeling of COVID-19 epidemic in the USA, UAE and Algeria. Alex. Eng. J. 60, 401-411 (2020)

Publisher's Note Springer Nature remains neutral with regard to jurisdictional claims in published maps and institutional affiliations. 